International Journal of Algebra and Statistics

Volume 7: 1-2(2018), 94-112

DOI :10.20454/ijas.2018.1455
Published by Modern Science Publishers

Available at: http://www.m-sciences.com

\title{
An application of complex neutrosophic sets to the theory of groups
}

\author{
Muhammad Gulistan ${ }^{\mathrm{a}}$, Florentin Smarandache ${ }^{\mathrm{b}}$, Amir Abdullah $^{\mathrm{a}}$ \\ ${ }^{a}$ Department of Mathematics and Statistics, Hazara University, Mansehra, Pakistan. \\ ${ }^{b}$ Department of Mathematics, University of New Mexico, USA. \\ (Received: 8 July 2018; Accepted: 2 November 2018)
}

\begin{abstract}
In this article we introduce the concept of complex neutrosophic subgroups (normal subgroups). We define the notion of alpha-cut of complex neutrosophic set, give examples and study some of its related results. We also define the Cartesian product of complex neutrosophic subgroups. Furthermore, we introduce the concept of image and preimage of complex neutrosophic set and prove some of its properties.
\end{abstract}

\section{Introduction}

[1], In 1965, Zadeh presented the idea of a fuzzy set. [2], Atanassov's in 1986, initiated the notion of intuitionistic fuzzy set which is the generalization of a fuzzy set. Neutrosophic set was first proposed by Smarandache in 1999 [5], which is the generalization of fuzzy set and intuitionistic fuzzy set. Neutrosophic set is characterized by a truth membership function, an indeterminacy membership function and a falsity membership function. In 2002, the Ramot et al. [8], generalized the concept of fuzzy set and introduced the notion of complex fuzzy set. There are many researchers which have worked on complex fuzzy set for instance, Buckly [6], Nguyen et al. [7] and Zhang et al. [9]. In contrast, Ramot et al. [8] presented an innovative concept that is totally different from other researchers, in which the author extended the range of membership function to the unit circle in the complex plane, unlike the others who limited to. Furthermore to solve enigma they also added an extra term which is called phase term in translating human language to complex valued functions on physical terms and vice versa. Abd Uazeez et al. in 2012 [10], added the non-membership term to the idea of complex fuzzy set which is known as complex intuitionistic fuzzy sets, the range of values are extended to the unit circle in complex plan for both membership and nonmembership functions instead of [0, 1]. In 2016, Mumtaz Ali et al. [12], extended the concept of complex fuzzy set, complex intuitionistic fuzzy set, and introduced the concept of complex neutrosophic sets which is a collection of complex-valued truth membership function, complex-valued indeterminacy membership function and complex-valued falsity membership function. Further in 1971, Rosenfeld [3], applied the concept of fuzzy set to groups and introduced the concept of fuzzy groups. The author defined fuzzy subgroups and studied some of its related properties. Vildan and Halis in 2017 [13], extended the concept of fuzzy subgroups on the base of neutrosophic sets and initiated the notion of neutrosophic subgroups.

2010 Mathematics Subject Classification. 16 Y60.

Keywords. Complex fuzzy sets; Complex neutrosophic sets; Neutrosophic subgroups; Complex neutrosophic subgroups; Complex neutrosophic normal subgroups.

Email addresses: gulistanmath@hu.edu.pk (Muhammad Gulistan), fsmarandache@gmail.com (Florentin Smarandache), amirabdullahktk@gmail.com (Amir Abdullah) 
Due to the motivation and inspiration of the above discussion. In this paper we introduce the concept of a complex neutrosophic subgroups (normal subgroups). We have give examples and study some related results. We also study the concept of Cartesian product of complex neutrosophic subgroups, image and preimage of complex neutrosophic set and alpha-cut of complex neutrosophic set with the help of examples and prove some of its properties.

\section{Preliminaries}

Here in this part we gathered some basic helping materials.

Definition 2.1. [1] A function $f$ is defined from a universe $\mathcal{X}$ to a closed interval $[0,1]$ is called a fuzzy set,i.e., a mapping:

$$
f: X \longrightarrow[0,1]
$$

Definition 2.2. [8] A complex fuzzy set (CFS) $\mathbb{C}$ over the universe $\mathcal{X}$, is defined an object of the form:

$$
\mathbb{C}=\left\{\left(x, \mu_{\mathbb{C}}(x)\right): x \in \mathcal{X}\right\}
$$

where $\mu_{\mathbb{C}}(x)=r_{\mathbb{C}}(x) \cdot e^{i \omega_{\mathbb{C}}(x)}$, here the amplitude term $r_{\mathbb{C}}(x)$ and phase term $\omega_{\mathbb{C}}(x)$, are real valued functions, for every $x \in \mathcal{X}$, the amplitude term $\mu_{\mathbb{C}}(x): \mathcal{X} \rightarrow[0,1]$ and phase term $\omega_{\mathbb{C}}(x)$ lying in the interval $[0,2 \pi]$.

Definition 2.3. [11] Let $\mathbb{C}_{1}$ and $\mathbb{C}_{2}$ be any two complex Atanassov's intuitionistic fuzzy sets (CAIFSs) over the universe $\mathcal{X}$, where

$$
\mathbb{C}_{1}=\left\{\left\langle x, r_{\mathbb{C}_{1}}(x) \cdot e^{i v_{\mathbb{C}_{1}}(x)}, k_{\mathbb{C}_{1}}(x) \cdot e^{i \omega_{\mathbb{C}_{1}}(x)}\right\rangle: x \in \mathcal{X}\right\}
$$

and

$$
\mathbb{C}_{2}=\left\{\left\langle x, r_{\mathbb{C}_{2}}(x) \cdot e^{i v_{\mathbb{C}_{2}}(x)}, k_{\mathbb{C}_{2}}(x) \cdot e^{i \omega_{\mathbb{C}_{2}}(x)}\right\rangle: x \in \mathcal{X}\right\} .
$$

Then

\section{Containment:}

$\mathbb{C}_{1} \subseteq \mathbb{C}_{2} \Leftrightarrow r_{\mathbb{C}_{1}}(x) \leq r_{\mathbb{C}_{2}}(x), k_{\mathbb{C}_{1}}(x) \geq k_{\mathbb{C}_{2}}(x)$ and $v_{\mathbb{C}_{1}}(x) \leq v_{\mathbb{C}_{2}}(x), \omega_{\mathbb{C}_{1}}(x) \geq \omega_{\mathbb{C}_{2}}(x)$.

\section{Equal:}

$$
\mathbb{C}_{1}=\mathbb{C}_{2} \Leftrightarrow r_{\mathbb{C}_{1}}(x)=r_{\mathbb{C}_{2}}(x), k_{\mathbb{C}_{1}}(x)=k_{\mathbb{C}_{2}}(x) \text { and } v_{\mathbb{C}_{1}}(x)=v_{\mathbb{C}_{2}}(x), \omega_{\mathbb{C}_{1}}(x)=\omega_{\mathbb{C}_{2}}(x) .
$$

Definition 2.4. [12] Let $\mathcal{X}$ be a universe of discourse, and $x \in \mathcal{X}$. A complex neutrosophic set (CNS) $\mathbb{C}$ in $\mathcal{X}$ is characterized by a complex truth membership function $\mathbb{C}_{T}(x)=p_{\mathbb{C}}(x) \cdot e^{i \mu_{\mathbb{C}}(x)}$, a complex indeterminacy membership function $\mathbb{C}_{I}(x)=q_{\mathbb{C}}(x) \cdot e^{i v_{\mathbb{C}}(x)}$ and a complex falsity membership function $\mathbb{C}_{F}(x)=r_{\mathbb{C}}(x) \cdot e^{i \omega_{\mathbb{C}}(x)}$. The values $\mathbb{C}_{T}(x), \mathbb{C}_{I}(x), \mathbb{C}_{F}(x)$ may lies all within the unit circle in the complex plane, where $p_{\mathbb{C}}(x), q_{\mathbb{C}}(x), r_{\mathbb{C}}(x)$ and $\mu_{\mathbb{C}}(x)$, $v_{\mathbb{C}}(x) \omega_{\mathbb{C}}(x)$ are amplitude terms and phase terms, respectively, and where $p_{\mathbb{C}}(x), q_{\mathbb{C}}(x), r_{\mathbb{C}}(x) \in[0,1]$, such that, $0 \leq p_{\mathbb{C}}(x)+q_{\mathbb{C}}(x)+r_{\mathbb{C}}(x) \leq 3$ and $\mu_{\mathbb{C}}(x), v_{\mathbb{C}}(x) \omega_{\mathbb{C}}(x) \in[0,2 \pi]$.

The complex neutrosophic set can be represented in the form as:

$$
\left.\mathbb{C}=\left\{\begin{array}{c}
x, \mathbb{C}_{T}(x)=p_{\mathbb{C}}(x) \cdot e^{i \mu_{\mathbb{C}}(x)}, \mathbb{C}_{I}(x)=q_{\mathbb{C}}(x) \cdot e^{i v_{\mathbb{C}}(x)}, \\
\mathbb{C}_{F}(x)=r_{\mathbb{C}}(x) \cdot e^{i \omega_{\mathbb{C}}(x)}
\end{array}\right): x \in \mathcal{X}\right\} .
$$

Example 2.5. Let $\mathcal{X}=\left\{x_{1}, x_{2}, x_{3}\right\}$ be the universe set and $\mathbb{C}$ be a complex neutrosophic set which is given by:

$$
\mathbb{C}=\left\{\begin{array}{c}
\left\langle x_{1}, 0.2 e^{0.5 \pi i}, 0.3 e^{0.6 \pi i}, 0.4 e^{0.8 \pi i}\right\rangle,\left\langle x_{2}, 0.4 e^{0.6 \pi i}, 0.5 e^{1.3 \pi i}, 0.1 e^{0.6 \pi i}\right\rangle, \\
\left\langle x_{3}, 0.1 e^{0.6 \pi i}, 0.3 e^{0.9 \pi i}, 0.9 e^{0.7 \pi i}\right\rangle
\end{array}\right\} .
$$


Definition 2.6. [3] Let $\mathcal{G}$ be any group with multiplication and $\mathcal{F}$ be a fuzzy subset of a group $\mathcal{G}$, then $\mathcal{F}$ is called a fuzzy subgroup (FSG) of $\mathcal{G}$, if the following axioms are hold:

(FSG1): $\mathcal{F}(x \cdot y) \geq \min \{\mathcal{F}(x), \mathcal{F}(y)\}$.

(FSG2): $\mathcal{F}\left(x^{-1}\right) \geq \mathcal{F}(x), \forall x, y \in \mathcal{G}$.

Definition 2.7. [13] Let $\mathcal{G}$ be any group with multiplication and $\mathcal{N}$ be a neutrosophic set on a group $\mathcal{G}$. Then $\mathcal{N}$ is called a neutrosophic subgroup (NSG) of $\mathcal{G}$, if its satisfy the following conditions:

(NSG1): $\mathcal{N}(x \cdot y) \geq \mathcal{N}(x) \wedge \mathcal{N}(y)$, i.e.,

$$
T_{\mathcal{N}}(x \cdot y) \geq T_{\mathcal{N}}(x) \wedge T_{\mathcal{N}}(y), I_{\mathcal{N}}(x \cdot y) \geq I_{\mathcal{N}}(x) \wedge I_{\mathcal{N}}(y) \text { and } F_{\mathcal{N}}(x \cdot y) \leq F_{\mathcal{N}}(x) \vee F_{\mathcal{N}}(y) .
$$

(NSG2): $\mathcal{N}\left(x^{-1}\right) \geq \mathcal{N}(x)$, i.e.,

$$
T_{\mathcal{N}}\left(x^{-1}\right) \geq T_{\mathcal{N}}(x), I_{\mathcal{N}}\left(x^{-1}\right) \geq I_{\mathcal{N}}(x) \text { and } F_{\mathcal{N}}\left(x^{-1}\right) \leq F_{\mathcal{N}}(x) \text {, for all } x \text { and } y \text { in } \mathcal{G} \text {. }
$$

\section{Complex Neutrosophic Subgroup}

Note: It should be noted that through out in this section we use a capital letter $\mathbb{C}$ to denote a complex neutrosophic set:

$$
\mathbb{C}=\left\{\left\langle T_{\mathbb{C}}=p_{\mathbb{C}} \cdot e^{i \mu_{\mathbb{C}}}, I_{\mathbb{C}}=q_{\mathbb{C}} \cdot e^{i v_{\mathbb{C}}}, F_{\mathbb{C}}=r_{\mathbb{C}} \cdot e^{i \omega_{\mathbb{C}}}\right\rangle\right\}
$$

Definition 3.1. A complex neutrosophic set $\mathbb{C}=\left\{\left\langle T_{\mathbb{C}}=p_{\mathbb{C}} \cdot e^{i \mu_{\mathbb{C}}}, I_{\mathbb{C}}=q_{\mathbb{C}} \cdot e^{i v_{\mathbb{C}}}, F_{\mathbb{C}}=r_{\mathbb{C}} \cdot e^{i \omega_{\mathbb{C}}}\right\rangle\right\}$ on a group $(\mathcal{G}, \cdot)$ is known as a complex neutrosophic subgroup (CNSG) of $\mathcal{G}$, if for all elements $x, y \in \mathcal{G}$, the following conditions are satisfied:

(CNSG1): $\mathbb{C}(x y) \geq \min \{\mathbb{C}(x), \mathbb{C}(y)\}$ i.e.,

(i) $p_{\mathbb{C}}(x y) \cdot e^{i \mu_{\mathbb{C}}(x y)} \geq \min \left\{p_{\mathbb{C}}(x) \cdot e^{i \mu_{\mathbb{C}}(x)}, p_{\mathbb{C}}(y) \cdot e^{i \mu_{\mathbb{C}}(y)}\right\}$

(ii) $q_{\mathbb{C}}(x y) \cdot e^{i v_{\mathbb{C}}(x y)} \geq \min \left\{q_{\mathbb{C}}(x) \cdot e^{i v_{\mathbb{C}}(x)}, q_{\mathbb{C}}(y) \cdot e^{i v_{\mathbb{C}}(y)}\right\}$

(iii) $r_{\mathbb{C}}(x y) \cdot e^{i \omega_{\mathbb{C}}(x y)} \leq \max \left\{r_{\mathbb{C}}(x) \cdot e^{i \omega_{\mathbb{C}}(x)}, r_{\mathbb{C}}(y) \cdot e^{i \omega_{\mathbb{C}}(y)}\right\}$

(CNSG2): $\mathbb{C}\left(x^{-1}\right) \geq \mathbb{C}(x)$ i.e.,

(iv) $p_{\mathbb{C}}\left(x^{-1}\right) \cdot e^{i \mu_{\mathbb{C}}\left(x^{-1}\right)} \geq p_{\mathbb{C}}(x) \cdot e^{i \mu_{\mathbb{C}}(x)}$

(v) $q_{\mathbb{C}}\left(x^{-1}\right) \cdot e^{i v_{\mathbb{C}}\left(x^{-1}\right)} \geq q_{\mathbb{C}}(x) \cdot e^{i v_{\mathbb{C}}(x)}$

(vi) $r_{\mathbb{C}}\left(x^{-1}\right) \cdot e^{i \omega_{\mathbb{C}}\left(x^{-1}\right)} \leq r_{\mathbb{C}}(x) \cdot e^{i \omega_{\mathbb{C}}(x)}$.

Example 3.2. Let $\mathcal{G}=\{1,-1, i,-i\}$ be a group under multiplication, and

$$
\mathbb{C}=\left\{\begin{array}{c}
\left\langle 1,0.7 e^{0.6 \pi i}, 0.6 e^{0.5 \pi i}, 0.5 e^{0.2 \pi i}\right\rangle,\left\langle-1,0.6 e^{0.5 \pi i}, 0.5 e^{0.4 \pi i}, 0.4 e^{0.2 \pi i}\right\rangle, \\
\left\langle i, 0.5 e^{0.3 \pi i}, 0.4 e^{0.2 \pi i}, 0.1 e^{0.2 \pi i}\right\rangle,\left\langle-i, 0.5 e^{0.3 \pi i}, 0.4 e^{0.2 \pi i}, 0.1 e^{0.2 \pi i}\right\rangle
\end{array}\right\}
$$

be a complex neutrosophic set on $\mathcal{G}$. Clearly $\mathbb{C}$ is a complex neutrosophic subgroup of $\mathcal{G}$. 


\subsection{Cartesian Product of Complex Neutrosophic Subgroups}

Definition 3.3. Let $\mathbb{C}_{1}=\left\langle\mathbb{C}_{1 T}(x), \mathbb{C}_{1 I}(x), \mathbb{C}_{1 F}(x)\right\rangle$ and $\mathbb{C}_{2}=\left\langle\mathbb{C}_{2 T}(x), \mathbb{C}_{2 I}(x), \mathbb{C}_{2 F}(x)\right\rangle$ be any two complex neutrosophic subgroups of the groups $\mathcal{G}_{1}$ and $\mathcal{G}_{2}$, respectively. Then the Cartesian product of $\mathbb{C}_{1}$ and $\mathbb{C}_{2}$, represented by $\mathbb{C}_{1} \times \mathbb{C}_{2}$ and define as:

$$
\mathbb{C}_{1} \times \mathbb{C}_{2}=\left\{\begin{array}{c}
\left\langle(x, y),\left(\mathbb{C}_{1} \times \mathbb{C}_{2}\right)_{T}(x, y),\left(\mathbb{C}_{1} \times \mathbb{C}_{2}\right)_{I}(x, y),\left(\mathbb{C}_{1} \times \mathbb{C}_{2}\right)_{F}(x, y)\right\rangle \\
/ \forall x \in \mathcal{G}_{1}, y \in \mathcal{G}_{2}
\end{array}\right\}
$$

where

$$
\begin{aligned}
& \left(\mathbb{C}_{1} \times \mathbb{C}_{2}\right)_{T}(x, y)=\min \left\{\mathbb{C}_{1 T}(x), \mathbb{C}_{2 T}(y)\right\}, \\
& \left(\mathbb{C}_{1} \times \mathbb{C}_{2}\right)_{I}(x, y)=\min \left\{\mathbb{C}_{1 I}(x), \mathbb{C}_{2 I}(y)\right\}, \\
& \left(\mathbb{C}_{1} \times \mathbb{C}_{2}\right)_{F}(x, y)=\max \left\{\mathbb{C}_{1 F}(x), \mathbb{C}_{2 F}(y)\right\} .
\end{aligned}
$$

Example 3.4. Let $\mathcal{G}_{1}=\{1,-1, i,-i\}$ and $\mathcal{G}_{2}=\left\{1, \omega, \omega^{2}\right\}$ are two groups under multiplication.

Consider,

$$
\mathbb{C}_{1}=\left\{\begin{array}{c}
\left\langle 1,0.7 e^{0.6 \pi i}, 0.6 e^{0.5 \pi i}, 0.5 e^{0.2 \pi i}\right\rangle,\left\langle-1,0.6 e^{0.5 \pi i}, 0.5 e^{0.4 \pi i}, 0.4 e^{0.2 \pi i}\right\rangle, \\
\left\langle i, 0.5 e^{0.3 \pi i}, 0.4 e^{0.2 \pi i}, 0.1 e^{0.2 \pi i}\right\rangle,\left\langle-i, 0.5 e^{0.3 \pi i}, 0.4 e^{0.2 \pi i}, 0.1 e^{0.2 \pi i}\right\rangle
\end{array}\right\}
$$

and

$$
\mathbb{C}_{2}=\left\{\begin{array}{c}
\left\langle 1,0.8 e^{0.6 \pi i}, 0.6 e^{0.5 \pi i}, 0.3 e^{0.2 \pi i}\right\rangle,\left\langle\omega, 0.7 e^{0.6 \pi i}, 0.5 e^{0.4 \pi i}, 0.3 e^{0.2 \pi i}\right\rangle, \\
\left\langle\omega^{2}, 0.7 e^{0.6 \pi i}, 0.5 e^{0.4 \pi i}, 0.3 e^{0.2 \pi i}\right\rangle
\end{array}\right\}
$$

are two complex neutrosophic subgroups of $\mathcal{G}_{1}$ and $\mathcal{G}_{2}$, respectively.

Now let $x=1$ and $y=\omega$, then

$$
\begin{aligned}
\mathbb{C}_{1} \times \mathbb{C}_{2} & =\left\{\left\langle\left(\mathbb{C}_{1} \times \mathbb{C}_{2}\right)_{T}(1, \omega),\left(\mathbb{C}_{1} \times \mathbb{C}_{2}\right)_{I}(1, \omega),\left(\mathbb{C}_{1} \times \mathbb{C}_{2}\right)_{F}(1, \omega)\right\rangle, \ldots\right\} \\
& =\left\{\left\langle\min \left\{\mathbb{C}_{1 T}(1), \mathbb{C}_{2 T}(\omega)\right\}, \min \left\{\mathbb{C}_{1 I}(1), \mathbb{C}_{2 I}(\omega)\right\}, \max \left\{\mathbb{C}_{1 F}(1),\right.\right.\right. \\
& \left.\left.\left.\mathbb{C}_{2 F}(\omega)\right\}\right\rangle, \ldots\right\} \\
& =\left\{\left\langle\min \left\{0.7 e^{0.6 \pi i}, 0.7 e^{0.6 \pi i}\right\}, \min \left\{0.6 e^{0.5 \pi i}, 0.5 e^{0.4 \pi i}\right\}, \max \left\{0.5 e^{0.2 \pi i},\right.\right.\right. \\
& \left.\left.\left.0.3 e^{0.2 \pi i}\right\}\right\rangle, \ldots\right\} \\
& =\left\{\left\langle 0.7 e^{0.6 \pi i}, 0.5 e^{0.4 \pi i}, 0.5 e^{0.2 \pi i}\right\rangle, \ldots\right\} .
\end{aligned}
$$

Theorem 3.5. If $\mathbb{C}_{1}$ and $\mathbb{C}_{2}$ are any two complex neutrosophic subgroups of the groups $\mathcal{G}_{1}$ and $\mathcal{G}_{2}$ respectively, then $\mathbb{C}_{1} \times \mathbb{C}_{2}$ is a complex neutrosophic subgroup of $\mathcal{G}_{1} \times \mathcal{G}_{2}$.

Proof: Assume that $\mathbb{C}_{1}=\left\langle\mathbb{C}_{1 T}, \mathbb{C}_{1 I}, \mathbb{C}_{1 F}\right\rangle$ and $\mathbb{C}_{2}=\left\langle\mathbb{C}_{2 T}, \mathbb{C}_{2 I}, \mathbb{C}_{2 F}\right\rangle$ be any two complex neutrosophic subgroups of the groups $\mathcal{G}_{1}$ and $\mathcal{G}_{2}$, respectively. Let any arbitrary elements $x_{1}, x_{2} \in \mathcal{G}_{1}$ and $y_{1}, y_{2} \in \mathcal{G}_{2}$, then $\left(x_{1}, y_{1}\right),\left(x_{2}, y_{2}\right) \in \mathcal{G}_{1} \times \mathcal{G}_{2}$.

Consider,

$$
\begin{aligned}
\left(\mathbb{C}_{1} \times \mathbb{C}_{2}\right)_{T}\left(\left(x_{1}, y_{1}\right),\left(x_{2}, y_{2}\right)\right) & =\left(\mathbb{C}_{1} \times \mathbb{C}_{2}\right)_{T}\left(x_{1} x_{2}, y_{1} y_{2}\right) \\
& =\min \left\{\mathbb{C}_{1 T}\left(x_{1} x_{2}\right), \mathbb{C}_{2 T}\left(y_{1} y_{2}\right)\right\} \\
& \geq \mathbb{C}_{1 T}\left(x_{1}\right) \wedge \mathbb{C}_{1 T}\left(x_{2}\right) \wedge \mathbb{C}_{2 T}\left(y_{1}\right) \wedge \mathbb{C}_{2 T}\left(y_{2}\right) \\
& =\mathbb{C}_{1 T}\left(x_{1}\right) \wedge \mathbb{C}_{2 T}\left(y_{1}\right) \wedge \mathbb{C}_{1 T}\left(x_{2}\right) \wedge \mathbb{C}_{2 T}\left(y_{2}\right) \\
& =\left(\mathbb{C}_{1} \times \mathbb{C}_{2}\right)_{T}\left(x_{1}, y_{1}\right) \wedge\left(\mathbb{C}_{1} \times \mathbb{C}_{2}\right)_{T}\left(x_{2}, y_{2}\right) .
\end{aligned}
$$


Similarly,

$$
\left(\mathbb{C}_{1} \times \mathbb{C}_{2}\right)_{I}\left(\left(x_{1}, y_{1}\right),\left(x_{2}, y_{2}\right)\right) \geq\left(\mathbb{C}_{1} \times \mathbb{C}_{2}\right)_{I}\left(x_{1}, y_{1}\right) \wedge\left(\mathbb{C}_{1} \times \mathbb{C}_{2}\right)_{I}\left(x_{2}, y_{2}\right),
$$

and

$$
\begin{aligned}
\left(\mathbb{C}_{1} \times \mathbb{C}_{2}\right)_{F}\left(\left(x_{1}, y_{1}\right),\left(x_{2}, y_{2}\right)\right) & =\left(\mathbb{C}_{1} \times \mathbb{C}_{2}\right)_{F}\left(x_{1} x_{2}, y_{1} y_{2}\right) \\
& =\max \left\{\mathbb{C}_{1 F}\left(x_{1} x_{2}\right), \mathbb{C}_{2 F}\left(y_{1} y_{2}\right)\right\} \\
& \leq \mathbb{C}_{1 F}\left(x_{1}\right) \vee \mathbb{C}_{1 F}\left(x_{2}\right) \vee \mathbb{C}_{2 F}\left(y_{1}\right) \vee \mathbb{C}_{2 F}\left(y_{2}\right) \\
& =\mathbb{C}_{1 F}\left(x_{1}\right) \vee \mathbb{C}_{2 F}\left(y_{1}\right) \vee \mathbb{C}_{1 F}\left(x_{2}\right) \vee \mathbb{C}_{2 F}\left(y_{2}\right) \\
& =\left(\mathbb{C}_{1} \times \mathbb{C}_{2}\right)_{F}\left(x_{1}, y_{1}\right) \vee\left(\mathbb{C}_{1} \times \mathbb{C}_{2}\right)_{F}\left(x_{2}, y_{2}\right) .
\end{aligned}
$$

Also,

$$
\begin{aligned}
\left(\mathbb{C}_{1} \times \mathbb{C}_{2}\right)_{T}\left(x_{1}, y_{1}\right)^{-1} & =\left(\mathbb{C}_{1} \times \mathbb{C}_{2}\right)_{T}\left(x_{1}^{-1}, y_{1}^{-1}\right) \\
& =\mathbb{C}_{1 T}\left(x_{1}^{-1}\right) \wedge \mathbb{C}_{2 T}\left(y_{1}^{-1}\right) \\
& \geq \mathbb{C}_{1 T}(x) \wedge \mathbb{C}_{2 T}(y) \\
& =\left(\mathbb{C}_{1} \times \mathbb{C}_{2}\right)_{T}(x, y) .
\end{aligned}
$$

Similarly,

$$
\left(\mathbb{C}_{1} \times \mathbb{C}_{2}\right)_{I}\left(x_{1}, y_{1}\right)^{-1} \geq\left(\mathbb{C}_{1} \times \mathbb{C}_{2}\right)_{I}(x, y) .
$$

And

$$
\begin{aligned}
\left(\mathbb{C}_{1} \times \mathbb{C}_{2}\right)_{F}\left(x_{1}, y_{1}\right)^{-1} & =\left(\mathbb{C}_{1} \times \mathbb{C}_{2}\right)_{F}\left(x_{1}^{-1}, y_{1}^{-1}\right) \\
& =\mathbb{C}_{1 F}\left(x_{1}^{-1}\right) \vee \mathbb{C}_{2 F}\left(y_{1}^{-1}\right) \\
& \leq \mathbb{C}_{1 F}(x) \vee \mathbb{C}_{2 F}(y) \\
& =\left(\mathbb{C}_{1} \times \mathbb{C}_{2}\right)_{F}(x, y) .
\end{aligned}
$$

Hence $\mathbb{C}_{1} \times \mathbb{C}_{2}$ is a complex neutrosophic subgroup of $\mathcal{G}_{1} \times \mathcal{G}_{2}$.

Theorem 3.6. Let $\mathbb{C}$ be a $C N S G$ of a group $\mathcal{G}$. Then the following properties are satisfied:

(a) $\mathbb{C}(\hat{e}) \cdot e^{i \mathbb{C}(\hat{e})} \geq \mathbb{C}(x) \cdot e^{i \mathbb{C}(x)} \forall x \in \mathcal{G}$, where $\hat{e}$ is the unit element of $\mathcal{G}$.

(b) $\mathbb{C}\left(x^{-1}\right) \cdot e^{i \mathbb{C}\left(x^{-1}\right)}=\mathbb{C}(x) \cdot e^{i \mathbb{C}(x)}$ for each $x \in \mathcal{G}$.

Proof: (a) Let $\hat{e}$ be the unit element of $\mathcal{G}$ and $x \in \mathcal{G}$ be arbitrary element, then by (CNSG1), (CNSG2) of Definition 3.1,

$$
\begin{aligned}
p_{\mathbb{C}}(\hat{e}) \cdot e^{i \mu_{\mathbb{C}}(\hat{e})} & =p_{\mathbb{C}}\left(x \cdot x^{-1}\right) \cdot e^{i \mu_{\mathbb{C}}\left(x \cdot x^{-1}\right)} \\
& \geq p_{\mathbb{C}}(x) \cdot e^{i \mu_{\mathbb{C}}(x)} \wedge p_{\mathbb{C}}\left(x^{-1}\right) \cdot e^{i \mu_{\mathbb{C}}\left(x^{-1}\right)} \\
& =p_{\mathbb{C}}(x) \cdot e^{i \mu_{\mathbb{C}}(x)} \wedge p_{\mathbb{C}}(x) \cdot e^{i \mu_{\mathbb{C}}(x)} \\
& =p_{\mathbb{C}}(x) \cdot e^{i \mu_{\mathbb{C}}(x)} \\
p_{\mathbb{C}}(\hat{e}) \cdot e^{i \mu_{\mathbb{C}}(\hat{e})} & \geq p_{\mathbb{C}}(x) \cdot e^{i \mu_{\mathbb{C}}(x)},
\end{aligned}
$$

Similarly,

$$
q_{\mathbb{C}}(\hat{e}) \cdot e^{i v_{\mathbb{C}}(\hat{e})} \geq q_{\mathbb{C}}(x) \cdot e^{i v_{\mathbb{C}}(x)} .
$$


And

$$
\begin{aligned}
r_{\mathbb{C}}(\hat{e}) \cdot e^{i \omega_{\mathbb{C}}(\hat{e})} & =r_{\mathbb{C}}\left(x \cdot x^{-1}\right) \cdot e^{i \omega_{\mathbb{C}}\left(x \cdot x^{-1}\right)} \\
& \leq r_{\mathbb{C}}(x) \cdot e^{i \omega_{\mathbb{C}}(x)} \vee r_{\mathbb{C}}\left(x^{-1}\right) \cdot e^{i \omega_{\mathbb{C}}\left(x^{-1}\right)} \\
& =r_{\mathbb{C}}(x) \cdot e^{i \omega_{\mathbb{C}}(x)} \vee r_{\mathbb{C}}(x) \cdot e^{i \omega_{\mathbb{C}}(x)} \\
& =r_{\mathbb{C}}(x) \cdot e^{i \omega_{\mathbb{C}}(x)} \\
r_{\mathbb{C}}(\hat{e}) \cdot e^{i \omega_{\mathbb{C}}(\hat{e})} & \leq r_{\mathbb{C}}(x) \cdot e^{i \omega_{\mathbb{C}}(x)}
\end{aligned}
$$

Hence $\mathbb{C}(\hat{e}) \cdot e^{i \mathbb{C}(\hat{e})} \geq \mathbb{C}(x) \cdot e^{i \mathbb{C}(x)}$ is satisfied, for all $x \in \mathcal{G}$.

(b) Let $x \in \mathcal{G}$. Since $\mathbb{C}$ is a complex neutrosophic subgroup of $\mathcal{G}$,

so $\mathbb{C}\left(x^{-1}\right) \cdot e^{i \mathbb{C}\left(x^{-1}\right)} \geq \mathbb{C}(x) \cdot e^{i \mathbb{C}(x)}$ is clear from (CNSG2) of Definition 3.1.

Again by applying (CNSG2) of Definition 3.1, and using group structure of $\mathcal{G}$, the other side of the inequality is proved as follows;

$$
\begin{aligned}
& p_{\mathbb{C}}(x) \cdot e^{i \mu_{\mathbb{C}}(x)}=p_{\mathbb{C}}\left(x^{-1}\right)^{-1} \cdot e^{i \mu_{\mathbb{C}}\left(x^{-1}\right)^{-1}} \geq p_{\mathbb{C}}\left(x^{-1}\right) \cdot e^{i \mu_{\mathbb{C}}\left(x^{-1}\right)}, \\
& q_{\mathbb{C}}(x) \cdot e^{i v_{\mathbb{C}}(x)}=q_{\mathbb{C}}\left(x^{-1}\right)^{-1} \cdot e^{i v_{\mathbb{C}}\left(x^{-1}\right)^{-1}} \geq q_{\mathbb{C}}\left(x^{-1}\right) \cdot e^{i v_{\mathbb{C}}\left(x^{-1}\right)}, \\
& r_{\mathbb{C}}(x) \cdot e^{i \omega_{\mathbb{C}}(x)}=r_{\mathbb{C}}\left(x^{-1}\right)^{-1} \cdot e^{i \omega_{\mathbb{C}}\left(x^{-1}\right)^{-1}} \leq r_{\mathbb{C}}\left(x^{-1}\right) \cdot e^{i \omega_{\mathbb{C}}\left(x^{-1}\right)} .
\end{aligned}
$$

Therefore,

$$
\mathbb{C}(x) \cdot e^{i \mathbb{C}(x)} \geq \mathbb{C}\left(x^{-1}\right) \cdot e^{i \mathbb{C}\left(x^{-1}\right)} .
$$

Thus,

$$
\mathbb{C}\left(x^{-1}\right) \cdot e^{i \mathbb{C}\left(x^{-1}\right)}=\mathbb{C}(x) \cdot e^{i \mathbb{C}(x)} .
$$

Hence $\mathbb{C}\left(x^{-1}\right) \cdot e^{i \mathbb{C}\left(x^{-1}\right)}=\mathbb{C}(x) \cdot e^{i \mathbb{C}(x)}$ is satisfied, for all $x \in \mathcal{G}$.

Theorem 3.7. Let $\mathbb{C}$ be a complex neutrosophic set on a group $\mathcal{G}$. Then $\mathbb{C}$ is a CNSG of $\mathcal{G}$ if and only if $\mathbb{C}\left(x \cdot y^{-1}\right)$. $e^{i \mathbb{C}\left(x \cdot y^{-1}\right)} \geq \mathbb{C}(x) \cdot e^{i \mathbb{C}(x)} \wedge \mathbb{C}(y) \cdot e^{i \mathbb{C}(y)}$ for each $x, y \in \mathcal{G}$.

Proof: Let $\mathbb{C}$ be a complex neutrosophic subgroup of $\mathcal{G}$ and $x, y \in \mathcal{G}$, So, it is clear that,

$$
\begin{aligned}
p_{\mathbb{C}}\left(x y^{-1}\right) \cdot e^{i \mu_{\mathbb{C}}\left(x y^{-1}\right)} & \geq p_{\mathbb{C}}(x) \cdot e^{i \mu_{\mathbb{C}}(x)} \wedge p_{\mathbb{C}}\left(y^{-1}\right) \cdot e^{i \mu_{\mathbb{C}}\left(y^{-1}\right)} \\
& \geq p_{\mathbb{C}}(x) \cdot e^{i \mu_{\mathbb{C}}(x)} \wedge p_{\mathbb{C}}(y) \cdot e^{i \mu_{\mathbb{C}}(y)}
\end{aligned}
$$

Similarly,

$$
q_{\mathbb{C}}\left(x y^{-1}\right) \cdot e^{i v_{\mathbb{C}}\left(x y^{-1}\right)} \geq q_{\mathbb{C}}(x) \cdot e^{i v_{\mathbb{C}}(x)} \wedge q_{\mathbb{C}}(y) \cdot e^{i v_{\mathbb{C}}(y)}
$$

And

$$
\begin{aligned}
r_{\mathbb{C}}\left(x y^{-1}\right) \cdot e^{i \omega_{\mathbb{C}}\left(x y^{-1}\right)} & \leq r_{\mathbb{C}}(x) \cdot e^{i \omega_{\mathbb{C}}(x)} \vee r_{\mathbb{C}}\left(y^{-1}\right) \cdot e^{i \omega_{\mathbb{C}}\left(y^{-1}\right)} \\
& \leq r_{\mathbb{C}}(x) \cdot e^{i \omega_{\mathbb{C}}(x)} \vee r_{\mathbb{C}}(y) \cdot e^{i \omega_{\mathbb{C}}(y)}
\end{aligned}
$$


Hence

$$
\begin{aligned}
\mathbb{C}\left(x \cdot y^{-1}\right) \cdot e^{i \mathbb{C}\left(x \cdot y^{-1}\right)} & =\left(p_{\mathbb{C}}\left(x y^{-1}\right) \cdot e^{i \mu_{\mathbb{C}}\left(x y^{-1}\right)}, q_{\mathbb{C}}\left(x y^{-1}\right) \cdot e^{i v_{\mathbb{C}}\left(x y^{-1}\right)},\right. \\
& \left.r_{\mathbb{C}}\left(x y^{-1}\right) \cdot e^{i \omega_{\mathbb{C}}\left(x y^{-1}\right)}\right) \\
& \geq\left(p_{\mathbb{C}}(x) \cdot e^{i \mu_{\mathbb{C}}(x)} \wedge p_{\mathbb{C}}(y) \cdot e^{i \mu_{\mathbb{C}}(y)}, q_{\mathbb{C}}(x) \cdot e^{i v_{\mathbb{C}}(x)}\right. \\
& \left.\wedge q_{\mathbb{C}}(y) \cdot e^{i v_{\mathbb{C}}(y)}, r_{\mathbb{C}}(x) \cdot e^{i \omega_{\mathbb{C}}(x)} \vee r_{\mathbb{C}}(y) \cdot e^{i \omega_{\mathbb{C}}(y)}\right) \\
& =\left(p_{\mathbb{C}}(x) \cdot e^{i \mu_{\mathbb{C}}(x)}, q_{\mathbb{C}}(x) \cdot e^{i v_{\mathbb{C}}(x)}, r_{\mathbb{C}}(x) \cdot e^{i \omega_{\mathbb{C}}(x)}\right) \\
& \wedge\left(p_{\mathbb{C}}(y) \cdot e^{i \mu_{\mathbb{C}}(y)}, q_{\mathbb{C}}(y) \cdot e^{i v_{\mathbb{C}}(y)}, r_{\mathbb{C}}(y) \cdot e^{i \omega_{\mathbb{C}}(y)}\right) \\
& =\mathbb{C}(x) \cdot e^{i \mathbb{C}(x)} \wedge \mathbb{C}(y) \cdot e^{i \mathbb{C}(y)} .
\end{aligned}
$$

Thus,

$$
\mathbb{C}\left(x \cdot y^{-1}\right) \cdot e^{i \mathbb{C}\left(x \cdot y^{-1}\right)} \geq \mathbb{C}(x) \cdot e^{i \mathbb{C}(x)} \wedge \mathbb{C}(y) \cdot e^{i \mathbb{C}(y)} .
$$

Conversely, Suppose the condition

$$
\mathbb{C}\left(x \cdot y^{-1}\right) \cdot e^{i \mathbb{C}\left(x \cdot y^{-1}\right)} \geq \mathbb{C}(x) \cdot e^{i \mathbb{C}(x)} \wedge \mathbb{C}(y) \cdot e^{i \mathbb{C}(y)}
$$

is hold.

Let $\hat{e}$ be the unit of $\mathcal{G}$, since $\mathcal{G}$ is a group,

$$
\begin{aligned}
p_{\mathbb{C}}\left(x^{-1}\right) \cdot e^{i \mu_{\mathbb{C}}\left(x^{-1}\right)} & =p_{\mathbb{C}}\left(\hat{e} \cdot x^{-1}\right) \cdot e^{i \mu_{\mathbb{C}}\left(\hat{e} \cdot x^{-1}\right)} \\
& \geq p_{\mathbb{C}}(\hat{e}) \cdot e^{i \mu_{\mathbb{C}}(\hat{e})} \wedge p_{\mathbb{C}}(x) \cdot e^{i \mu_{\mathbb{C}}(x)} \\
& =p_{\mathbb{C}}\left(x \cdot x^{-1}\right) \cdot e^{i \mu_{\mathbb{C}}\left(x \cdot x^{-1}\right)} \wedge p_{\mathbb{C}}(x) \cdot e^{i \mu_{\mathbb{C}}(x)} \\
& \geq p_{\mathbb{C}}(x) \cdot e^{i \mu_{\mathbb{C}}(x)} \wedge p_{\mathbb{C}}(x) \cdot e^{i \mu_{\mathbb{C}}(x)} \wedge p_{\mathbb{C}}(x) \cdot e^{i \mu_{\mathbb{C}}(x)} \\
& =p_{\mathbb{C}}(x) \cdot e^{i \mu_{\mathbb{C}}(x)} \\
p_{\mathbb{C}}\left(x^{-1}\right) \cdot e^{i \mu_{\mathbb{C}}\left(x^{-1}\right)} & \geq p_{\mathbb{C}}(x) \cdot e^{i \mu_{\mathbb{C}}(x)} .
\end{aligned}
$$

Similarly,

$$
q_{\mathbb{C}}\left(x^{-1}\right) \cdot e^{i v_{\mathbb{C}}\left(x^{-1}\right)} \geq q_{\mathbb{C}}(x) \cdot e^{i v_{\mathbb{C}}(x)}
$$

And

$$
\begin{aligned}
r_{\mathbb{C}}\left(x^{-1}\right) \cdot e^{i \omega_{\mathbb{C}}\left(x^{-1}\right)} & =r_{\mathbb{C}}\left(\hat{e} \cdot x^{-1}\right) \cdot e^{i \omega_{\mathbb{C}}\left(\hat{e} \cdot x^{-1}\right)} \\
& \leq r_{\mathbb{C}}(\hat{e}) \cdot e^{i \omega_{\mathbb{C}}(\hat{e})} \vee r_{\mathbb{C}}(x) \cdot e^{i \omega_{\mathbb{C}}(x)} \\
& =r_{\mathbb{C}}\left(x \cdot x^{-1}\right) \cdot e^{i \omega_{\mathbb{C}}\left(x \cdot x^{-1}\right)} \vee r_{\mathbb{C}}(x) \cdot e^{i \omega_{\mathbb{C}}(x)} \\
& \leq r_{\mathbb{C}}(x) \cdot e^{i \omega_{\mathbb{C}}(x)} \vee r_{\mathbb{C}}(x) \cdot e^{i \omega_{\mathbb{C}}(x)} \vee r_{\mathbb{C}}(x) \cdot e^{i \omega_{\mathbb{C}}(x)} \\
& =\vee r_{\mathbb{C}}(x) \cdot e^{i \omega_{\mathbb{C}}(x)}
\end{aligned}
$$

So, the condition (CNSG2) of Definition 3.1 is satisfied.

Now let us show the condition (CNSG1) of Definition 3.1,

$$
\begin{aligned}
p_{\mathbb{C}}(x \cdot y) \cdot e^{i \mu_{\mathbb{C}}(x \cdot y)} & =p_{\mathbb{C}}\left(x \cdot\left(y^{-1}\right)^{-1}\right) \cdot e^{i \mu_{\mathbb{C}}\left(x \cdot\left(y^{-1}\right)^{-1}\right)} \\
& \geq p_{\mathbb{C}}(x) \cdot e^{i \mu_{\mathbb{C}}(x)} \wedge p_{\mathbb{C}}\left(y^{-1}\right) \cdot e^{i \mu_{\mathbb{C}}\left(y^{-1}\right)} \\
& \geq p_{\mathbb{C}}(x) \cdot e^{i \mu_{\mathbb{C}}(x)} \wedge p_{\mathbb{C}}(y) \cdot e^{i \mu_{\mathbb{C}}(y)} .
\end{aligned}
$$


Similarly,

$$
q_{\mathbb{C}}(x \cdot y) \cdot e^{i v_{\mathbb{C}}(x \cdot y)} \geq q_{\mathbb{C}}(x) \cdot e^{i v_{\mathbb{C}}(x)} \wedge q_{\mathbb{C}}(y) \cdot e^{i v_{\mathbb{C}}(y)}
$$

and

$$
\begin{aligned}
r_{\mathbb{C}}(x \cdot y) \cdot e^{i \omega_{\mathbb{C}}(x \cdot y)} & =r_{\mathbb{C}}\left(x \cdot\left(y^{-1}\right)^{-1}\right) \cdot e^{i \omega_{\mathbb{C}}\left(x \cdot\left(y^{-1}\right)^{-1}\right)} \\
& \leq r_{\mathbb{C}}(x) \cdot e^{i \omega_{\mathbb{C}}(x)} \vee r_{\mathbb{C}}\left(y^{-1}\right) \cdot e^{i \omega_{\mathbb{C}}\left(y^{-1}\right)} \\
& \leq r_{\mathbb{C}}(x) \cdot e^{i \omega_{\mathbb{C}}(x)} \vee r_{\mathbb{C}}(y) \cdot e^{i \omega_{\mathbb{C}}(y)} .
\end{aligned}
$$

Therefore (CNSG1) of Definition 3.1 is also satisfied. Thus $\mathbb{C}$ is a complex neutrosophic subgroup of $\mathcal{G}$.

Based on Theorem 3.7, we define complex neutrosophic subgroup as follows:

Definition 3.8. Let $\mathcal{G}$ be any group with multiplication. A complex neutrosophic set

$$
\mathbb{C}=\left\{\left\langle T_{\mathbb{C}}=p_{\mathbb{C}} \cdot e^{i \mu_{\mathbb{C}}}, I_{\mathbb{C}}=q_{\mathbb{C}} \cdot e^{i v_{\mathbb{C}}}, F_{\mathbb{C}}=r_{\mathbb{C}} \cdot e^{i \omega_{\mathbb{C}}}\right\rangle\right\}
$$

on group $\mathcal{G}$ is known as a complex neutrosophic subgroup (CNSG) of $\mathcal{G}$, if

$\mathbb{C}\left(x^{-1} y\right) \geq \min \{\mathbb{C}(x), \mathbb{C}(y)\}$ i.e.,

(i) $p_{\mathbb{C}}\left(x^{-1} y\right) \cdot e^{i \mu_{\mathbb{C}}\left(x^{-1} y\right)} \geq \min \left\{p_{\mathbb{C}}(x) \cdot e^{i \mu_{\mathbb{C}}(x)}, p_{\mathbb{C}}(y) \cdot e^{i \mu_{\mathbb{C}}(y)}\right\}$

(ii) $q_{\mathbb{C}}\left(x^{-1} y\right) \cdot e^{i v_{\mathbb{C}}\left(x^{-1} y\right)} \geq \min \left\{q_{\mathbb{C}}(x) \cdot e^{i v_{\mathbb{C}}(x)}, q_{\mathbb{C}}(y) \cdot e^{i v_{\mathbb{C}}(y)}\right\}$

(iii) $r_{\mathbb{C}}\left(x^{-1} y\right) \cdot e^{i \omega_{\mathbb{C}}\left(x^{-1} y\right)} \leq \max \left\{r_{\mathbb{C}}(x) \cdot e^{i \omega_{\mathbb{C}}(x)}, r_{\mathbb{C}}(y) \cdot e^{i \omega_{\mathbb{C}}(y)}\right\}, \forall x, y \in \mathcal{G}$.

Example 3.9. Let $\mathcal{G}=\{1,-1, i,-i\}$ be a group under multiplication, and $\mathbb{C}=\left\langle T_{\mathbb{C}}, I_{\mathbb{C}}, F_{\mathbb{C}}\right\rangle$ be complex neutrosophic set on $\mathcal{G}$, such that

$$
\begin{aligned}
& T_{\mathbb{C}}(1)=0.8 e^{0.6 \pi i}, T_{\mathbb{C}}(-1)=0.7 e^{0.5 \pi i}, T_{\mathbb{C}}(i)=T_{\mathbb{C}}(-i)=0.3 e^{0.2 \pi i} \\
& I_{\mathbb{C}}(1)=0.7 e^{0.5 \pi i}, I_{\mathbb{C}}(-1)=0.6 e^{0.4 \pi i}, I_{\mathbb{C}}(i)=I_{\mathbb{C}}(-i)=0.2 e^{0.2 \pi i} \\
& F_{\mathbb{C}}(1)=0.5 e^{0.4 \pi i}, F_{\mathbb{C}}(-1)=0.1 e^{0.2 \pi i}, F_{\mathbb{C}}(i)=F_{\mathbb{C}}(-i)=0.1 e^{0.2 \pi i} .
\end{aligned}
$$

Clearly, $\mathbb{C}$ is a complex neutrosophic subgroup of $\mathcal{G}$.

Theorem 3.10. If $\mathbb{C}_{1}$ and $\mathbb{C}_{2}$ are two complex neutrosophic subgroups of a group $\mathcal{G}$, then the intersection $\mathbb{C}_{1} \cap \mathbb{C}_{2}$ is a complex neutrosophic subgroup of $\mathcal{G}$.

Proof: Let $x, y \in \mathcal{G}$ be any arbitrary elements. By Theorem 3.7, it is enough to show that

$$
\left(\mathbb{C}_{1} \cap \mathbb{C}_{2}\right)\left(x \cdot y^{-1}\right) \geq\left(\mathbb{C}_{1} \cap \mathbb{C}_{2}\right)(x) \wedge\left(\mathbb{C}_{1} \cap \mathbb{C}_{2}\right)(y) .
$$

First consider the truth-membership degree of the intersection

$$
\begin{aligned}
p_{\mathbb{C}_{1} \cap \mathbb{C}_{2}}\left(x \cdot y^{-1}\right) \cdot e^{i \mu_{\mathbb{C}_{1} \cap \mathbb{C}_{2}}\left(x \cdot y^{-1}\right)} & =p_{\mathbb{C}_{1}}\left(x \cdot y^{-1}\right) \cdot e^{i \mu_{\mathbb{C}_{1}}\left(x \cdot y^{-1}\right)} \\
& \wedge p_{\mathbb{C}_{2}}\left(x \cdot y^{-1}\right) \cdot e^{i \mu_{\mathbb{C}_{2}}\left(x \cdot y^{-1}\right)} \\
& \geq p_{\mathbb{C}_{1}}(x) \cdot e^{i \mu_{\mathbb{C}_{1}}(x)} \wedge p_{\mathbb{C}_{1}}(y) \cdot e^{i \mu_{\mathbb{C}_{1}}(y)} \\
& \wedge p_{\mathbb{C}_{2}}(x) \cdot e^{i \mu_{\mathbb{C}_{2}}(x)} \wedge p_{\mathbb{C}_{2}}(y) \cdot e^{i \mu_{\mathbb{C}_{2}}(y)} \\
& =\left(p_{\mathbb{C}_{1}}(x) \cdot e^{i \mu_{\mathbb{C}_{1}}(x)} \wedge p_{\mathbb{C}_{2}}(x) \cdot e^{i \mu_{\mathbb{C}_{2}}(x)}\right) \\
& \wedge\left(p_{\mathbb{C}_{1}}(y) \cdot e^{i \mu_{\mathbb{C}_{1}}(y)} \wedge p_{\mathbb{C}_{2}}(y) \cdot e^{i \mu_{\mathbb{C}_{2}}(y)}\right) \\
& =p_{\mathbb{C}_{1} \cap \mathbb{C}_{2}}(x) \cdot e^{i \mu_{\mathbb{C}_{1} \cap \mathbb{C}_{2}}(x)} \\
& \wedge p_{\mathbb{C}_{1} \cap \mathbb{C}_{2}}(y) \cdot e^{i \mu_{\mathbb{C}_{1} \cap \mathbb{C}_{2}}(y)} .
\end{aligned}
$$


Similarly,

$$
\begin{aligned}
q_{\mathbb{C}_{1} \cap \mathbb{C}_{2}}\left(x \cdot y^{-1}\right) \cdot e^{i v_{\mathbb{C}_{1} \cap \mathbb{C}_{2}}\left(x \cdot y^{-1}\right)} & \geq q_{\mathbb{C}_{1} \cap \mathbb{C}_{2}}(x) \cdot e^{i v_{\mathbb{C}_{1} \cap \mathbb{C}_{2}}(x)} \\
& \wedge q_{\mathbb{C}_{1} \cap \mathbb{C}_{2}}(y) \cdot e^{i v_{\mathbb{C}_{1} \cap \mathbb{C}_{2}}(y) .} .
\end{aligned}
$$

And

$$
\begin{aligned}
r_{\mathbb{C}_{1} \cup \mathbb{C}_{2}}\left(x \cdot y^{-1}\right) \cdot e^{i \omega_{\mathbb{C}_{1}} \cup \mathbb{C}_{2}}\left(x \cdot y^{-1}\right) & =r_{\mathbb{C}_{1}}\left(x \cdot y^{-1}\right) \cdot e^{i \omega_{\mathbb{C}_{1}}\left(x \cdot y^{-1}\right)} \\
& \vee r_{\mathbb{C}_{2}}\left(x \cdot y^{-1}\right) \cdot e^{i \omega_{\mathbb{C}_{2}}\left(x \cdot y^{-1}\right)} \\
& \leq r_{\mathbb{C}_{1}}(x) \cdot e^{i \omega_{\mathbb{C}_{1}}(x)} \vee r_{\mathbb{C}_{1}}(y) \cdot e^{i \omega_{\mathbb{C}_{1}}(y)} \\
& \vee r_{\mathbb{C}_{2}}(x) \cdot e^{i \omega_{\mathbb{C}_{2}}(x)} \vee r_{\mathbb{C}_{2}}(y) \cdot e^{i \omega_{\mathbb{C}_{2}}(y)} \\
& =r_{\mathbb{C}_{1}}(x) \cdot e^{i \omega_{\mathbb{C}_{1}}(x)} \vee r_{\mathbb{C}_{2}}(x) \cdot e^{i \omega_{\mathbb{C}_{2}}(x)} \\
& \vee r_{\mathbb{C}_{1}}(y) \cdot e^{i \omega_{\mathbb{C}_{1}}(y)} \vee r_{\mathbb{C}_{2}}(y) \cdot e^{i \omega_{\mathbb{C}_{2}}(y)} \\
& =r_{\mathbb{C}_{1} \cup \mathbb{C}_{2}}(x) \cdot e^{i \omega \omega_{1} \cup \mathbb{C}_{2}}(x) \\
& \vee r_{\mathbb{C}_{1} \cup \mathbb{C}_{2}}(y) \cdot e^{i \omega \mathbb{C}_{1} \cup \mathbb{C}_{2}}(y) .
\end{aligned}
$$

Hence $\mathbb{C}_{1} \cap \mathbb{C}_{2}$ is a complex neutrosophic subgroup of $\mathcal{G}$.

Theorem 3.11. If $\mathbb{C}_{1}$ and $\mathbb{C}_{2}$ are two complex neutrosophic subgroups of a group $\mathcal{G}$, then the union $\mathbb{C}_{1} \cup \mathbb{C}_{2}$ is a complex neutrosophic subgroup of $\mathcal{G}$.

Proof: Let $x, y \in \mathcal{G}$ be any arbitrary elements. By Theorem 3.7, it is enough to show that

$\left(\mathbb{C}_{1} \cup \mathbb{C}_{2}\right)\left(x \cdot y^{-1}\right) \geq \min \left\{\left(\mathbb{C}_{1} \cup \mathbb{C}_{2}\right)(x),\left(\mathbb{C}_{1} \cup \mathbb{C}_{2}\right)(y)\right\}$.

Consider,

$$
\begin{aligned}
p_{\mathbb{C}_{1} \cup \mathbb{C}_{2}}\left(x \cdot y^{-1}\right) \cdot e^{i \mu_{\mathbb{C}_{1} \cup \mathbb{C}_{2}}\left(x \cdot y^{-1}\right)} & =p_{\mathbb{C}_{1}}\left(x \cdot y^{-1}\right) \cdot e^{i \mu_{\mathbb{C}_{1}}\left(x \cdot y^{-1}\right)} \\
& \vee p_{\mathbb{C}_{2}}\left(x \cdot y^{-1}\right) \cdot e^{i \mu_{\mathbb{C}_{2}}\left(x \cdot y^{-1}\right)} \\
& \geq p_{\mathbb{C}_{1}}(x) \cdot e^{i \mu_{\mathbb{C}_{1}}(x)} \wedge p_{\mathbb{C}_{1}}(y) \cdot e^{i \mu_{\mathbb{C}_{1}}(y)} \\
& \vee p_{\mathbb{C}_{2}}(x) \cdot e^{i \mu_{\mathbb{C}_{2}}(x)} \wedge p_{\mathbb{C}_{2}}(y) \cdot e^{i \mu_{\mathbb{C}_{2}}(y)} \\
& =\left(p_{\mathbb{C}_{1}}(x) \cdot e^{i \mu_{\mathbb{C}_{1}}(x)} \vee p_{\mathbb{C}_{2}}(x) \cdot e^{i \mu_{\mathbb{C}_{2}}(x)}\right) \\
& \wedge\left(p_{\mathbb{C}_{1}}(y) \cdot e^{i \mu_{\mathbb{C}_{1}}(y)} \vee p_{\mathbb{C}_{2}}(y) \cdot e^{i \mu_{\mathbb{C}_{2}}(y)}\right) \\
& =\min \left\{p_{\mathbb{C}_{1} \cup \mathbb{C}_{2}}(x) \cdot e^{i \mu_{\mathbb{C}_{1}} \cup \mathbb{C}_{2}}(x)\right. \\
& \left.p_{\mathbb{C}_{1} \cup \mathbb{C}_{2}}(y) \cdot e^{i \mu_{\mathbb{C}_{1} \cup \mathbb{C}_{2}}(y)}\right\} .
\end{aligned}
$$

And

$$
\begin{aligned}
r_{\mathbb{C}_{1} \cap \mathbb{C}_{2}}\left(x \cdot y^{-1}\right) \cdot e^{i \omega_{\mathbb{C}_{1} \cap \mathbb{C}_{2}}\left(x \cdot y^{-1}\right)} & =r_{\mathbb{C}_{1}}\left(x \cdot y^{-1}\right) \cdot e^{i \omega_{\mathbb{C}_{1}}\left(x \cdot y^{-1}\right)} \\
& \wedge r_{\mathbb{C}_{2}}\left(x \cdot y^{-1}\right) \cdot e^{i \omega_{\mathbb{C}_{2}}\left(x \cdot y^{-1}\right)} \\
& \leq r_{\mathbb{C}_{1}}(x) \cdot e^{i \omega_{\mathbb{C}_{1}}(x)} \vee r_{\mathbb{C}_{1}}(y) \cdot e^{i \omega_{\mathbb{C}_{1}}(y)} \\
& \wedge r_{\mathbb{C}_{2}}(x) \cdot e^{i \omega_{\mathbb{C}_{2}}(x)} \vee r_{\mathbb{C}_{2}}(y) \cdot e^{i \omega_{\mathbb{C}_{2}}(y)} \\
& =r_{\mathbb{C}_{1}}(x) \cdot e^{i \omega_{\mathbb{C}_{1}}(x)} \wedge r_{\mathbb{C}_{2}}(x) \cdot e^{i \omega_{\mathbb{C}_{2}}(x)} \\
& \vee r_{\mathbb{C}_{1}}(y) \cdot e^{i \omega_{\mathbb{C}_{1}}(y)} \wedge r_{\mathbb{C}_{2}}(y) \cdot e^{i \omega_{\mathbb{C}_{2}}(y)} \\
& =\max \left\{r_{\mathbb{C}_{1} \cap \mathbb{C}_{2}}(x) \cdot e^{i \omega \mathrm{C}_{1} \cap \mathbb{C}_{2}}(x),\right. \\
& \left.r_{\mathbb{C}_{1} \cap \mathbb{C}_{2}}(y) \cdot e^{i \omega_{\mathbb{C}_{1}} \cap \mathbb{C}_{2}}(y)\right\} .
\end{aligned}
$$

Thus, $\mathbb{C}_{1} \cup \mathbb{C}_{2}$ is a complex neutrosophic subgroup of $\mathcal{G}$. 


\section{Alpha-Cut of Complex Neutrosophic Set}

Definition 4.1. Let $\mathbb{C}=\left\langle\mathbb{C}_{T}=p_{\mathbb{C}} e^{i \mu_{\mathbb{C}}}, \mathbb{C}_{I}=q_{\mathbb{C}} e^{i v_{\mathbb{C}}}, \mathbb{C}_{F}=r_{\mathbb{C}} e^{i \omega_{\mathbb{C}}}\right\rangle$ be a complex neutrosophic set on $\mathcal{X}$ and $\alpha=\beta \cdot e^{i \gamma}$, where $\beta \in[0,1], \gamma \in[0,2 \pi]$.

Define the $\alpha$-level set of $\mathbb{C}$ as follows:

$\mathbb{C}_{\alpha}=\{x \in \mathcal{X} \mid \mathbb{C}(x) \geq \alpha\}$ i.e.

$$
\begin{aligned}
& \left(p_{\mathbb{C}}(x) \cdot e^{i \mu_{\mathbb{C}}(x)}\right)_{\alpha}=\left\{x \in \mathcal{X} \mid p_{\mathbb{C}}(x) \cdot e^{i \mu_{\mathbb{C}}(x)} \geq \beta \cdot e^{i \gamma}\right\}, \\
& \left(q_{\mathbb{C}}(x) \cdot e^{i v_{\mathbb{C}}(x)}\right)_{\alpha}=\left\{x \in \mathcal{X} \mid q_{\mathbb{C}}(x) \cdot e^{i v_{\mathbb{C}}(x)} \geq \beta \cdot e^{i \gamma}\right\}, \\
& \left(r_{\mathbb{C}}(x) \cdot e^{i \omega_{\mathbb{C}}(x)}\right)^{\alpha}=\left\{x \in \mathcal{X} \mid r_{\mathbb{C}}(x) \cdot e^{i \omega_{\mathbb{C}}(x)} \leq \beta \cdot e^{i \gamma}\right\} .
\end{aligned}
$$

It is easy to verify that,

(1) If $\mathbb{C}_{1} \subseteq \mathbb{C}_{2}$ and $\alpha=\beta \cdot e^{i \gamma}$, where, $\beta \in[0,1], \gamma \in[0,2 \pi]$, then,

$$
\begin{aligned}
& \left(p_{\mathbb{C}_{1}}(x) \cdot e^{i \mu_{\mathbb{C}_{1}}(x)}\right)_{\alpha} \subseteq\left(p_{\mathbb{C}_{2}}(x) \cdot e^{i \mu_{\mathbb{C}_{2}}(x)}\right)_{\alpha} \\
& \left(q_{\mathbb{C}_{1}}(x) \cdot e^{i v_{\mathbb{C}_{1}}(x)}\right)_{\alpha} \subseteq\left(q_{\mathbb{C}_{2}}(x) \cdot e^{i v_{\mathbb{C}_{2}}(x)}\right)_{\alpha} \\
& \left(r_{\mathbb{C}_{1}}(x) \cdot e^{i \omega_{\mathbb{C}_{1}}(x)}\right)^{\alpha} \supseteq\left(r_{\mathbb{C}_{2}}(x) \cdot e^{i \omega_{\mathbb{C}_{2}}(x)}\right)^{\alpha} .
\end{aligned}
$$

(2) $\alpha_{1} \leq \alpha_{2}$ where, $\alpha_{1}=\beta_{1} \cdot e^{i \gamma_{1}}, \alpha_{2}=\beta_{2} \cdot e^{i \gamma_{2}}$ implies that

$$
\begin{aligned}
& \left(p_{\mathbb{C}_{1}}(x) \cdot e^{i \mu_{\mathbb{C}_{1}}(x)}\right)_{\alpha_{1}} \supseteq\left(p_{\mathbb{C}_{1}}(x) \cdot e^{i \mu_{\mathbb{C}_{1}}(x)}\right)_{\alpha_{2}} \\
& \left(q_{\mathbb{C}_{1}}(x) \cdot e^{i v_{\mathbb{C}_{1}}(x)}\right)_{\alpha_{1}} \supseteq\left(q_{\mathbb{C}_{1}}(x) \cdot e^{i v_{\mathbb{C}_{1}}(x)}\right)_{\alpha_{2}} \\
& \left(r_{\mathbb{C}_{1}}(x) \cdot e^{i \omega_{\mathbb{C}_{1}}(x)}\right)^{\alpha_{1}} \subseteq\left(r_{\mathbb{C}_{1}}(x) \cdot e^{i \omega_{\mathbb{C}_{1}}(x)}\right)^{\alpha_{2}} .
\end{aligned}
$$

Example 4.2. Let

$$
\mathbb{C}=\left\{\begin{array}{c}
\left\langle x_{1}, 0.2 e^{0.4 \pi i}, 0.3 e^{0.5 \pi i}, 0.7 e^{0.1 \pi i}\right\rangle,\left\langle x_{2}, 0.7 e^{0.1 \pi i}, 0.6 e^{0.5 \pi i}, 0.7 e^{0.4 \pi i}\right\rangle, \\
\left\langle x_{3}, 0.6 e^{0.4 \pi i}, 0.4 e^{0.5 \pi i}, 0.1 e^{0.4 \pi i}\right\rangle
\end{array}\right\}
$$

be a complex neutrosophic set of $\mathcal{X}$, and $\alpha=0.4 e^{0.4 \pi i}$. Then the $\alpha$-level set as: $\mathbb{C}_{\alpha}=\left\{x_{3}\right\}$.

Proposition 4.3. $\mathbb{C}$ is a complex neutrosophic subgroup of $\mathcal{G}$ if and only if for all $\alpha=\beta e^{i \gamma}$ where, $\beta \in[0,1], \gamma \in$ $[0,2 \pi], \alpha$-level sets of $\mathbb{C},\left(p_{\mathbb{C}} \cdot e^{i \mu_{\mathbb{C}}}\right)_{\alpha},\left(q_{\mathbb{C}} \cdot e^{i v_{\mathbb{C}}}\right)_{\alpha}$ and $\left(r_{\mathbb{C}} \cdot e^{i \omega_{\mathbb{C}}}\right)^{\alpha}$ are classical subgroups of $\mathcal{G}$.

Proof: Let $\mathbb{C}$ be a CNSG of $\mathcal{G}, \alpha=\beta e^{i \gamma}$ where $\beta \in[0,1], \gamma \in[0,2 \pi]$ and $x, y \in\left(p_{\mathbb{C}} \cdot e^{i \mu_{\mathbb{C}}}\right)_{\alpha}$ (similarly $\left.x, y \in\left(q_{\mathbb{C}} \cdot e^{i v_{\mathbb{C}}}\right)_{\alpha},\left(r_{\mathbb{C}} \cdot e^{i \omega_{\mathbb{C}}}\right)^{\alpha}\right)$.

By the assumption,

$$
\begin{aligned}
p_{\mathbb{C}}\left(x \cdot y^{-1}\right) \cdot e^{i \mu_{\mathbb{C}}\left(x \cdot y^{-1}\right)} & \geq p_{\mathbb{C}}(x) \cdot e^{i \mu_{\mathbb{C}}(x)} \wedge p_{\mathbb{C}}(y) \cdot e^{i \mu_{\mathbb{C}}(y)} \\
& \geq \alpha \wedge \alpha=\alpha .
\end{aligned}
$$

Similarly,

$$
q_{\mathbb{C}}\left(x \cdot y^{-1}\right) \cdot e^{i v_{\mathbb{C}}\left(x \cdot y^{-1}\right)} \geq \alpha .
$$

And

$$
\begin{aligned}
r_{\mathbb{C}}\left(x \cdot y^{-1}\right) \cdot e^{i \omega_{\mathbb{C}}\left(x \cdot y^{-1}\right)} & \leq r_{\mathbb{C}}(x) \cdot e^{i \omega_{\mathbb{C}}(x)} \vee r_{\mathbb{C}}(y) \cdot e^{i \omega_{\mathbb{C}}(y)} \\
& \leq \alpha \vee \alpha=\alpha
\end{aligned}
$$


Hence $x \cdot y^{-1} \in\left(p_{\mathbb{C}} \cdot e^{i \mu_{\mathbb{C}}}\right)_{\alpha},\left(q_{\mathbb{C}} \cdot e^{i v_{\mathbb{C}}}\right)_{\alpha},\left(r_{\mathbb{C}} \cdot e^{i \omega_{\mathbb{C}}}\right)^{\alpha}$ for each $\alpha$.

This means that $\left(p_{\mathbb{C}}(x) \cdot e^{i \mu_{\mathbb{C}}(x)}\right)_{\alpha},\left(q_{\mathbb{C}}(x) \cdot e^{i v_{\mathbb{C}}(x)}\right)_{\alpha}$ and $\left(r_{\mathbb{C}}(x) \cdot e^{i \omega_{\mathbb{C}}(x)}\right)^{\alpha}$ is a classical subgroup of $\mathcal{G}$ for each $\alpha$.

Conversely, let $\left(p_{\mathbb{C}} \cdot e^{i \mu_{\mathbb{C}}}\right)_{\alpha}$ be a classical subgroup of $\mathcal{G}$, for each $\alpha=\beta e^{i \gamma}$ where $\beta \in[0,1], \gamma \in[0,2 \pi]$.

Let $x, y \in \mathcal{G}, \alpha=p_{\mathbb{C}}(x) \cdot e^{i \mu_{\mathbb{C}}(x)} \wedge p_{\mathbb{C}}(y) \cdot e^{i \mu_{\mathbb{C}}(y)}$ and $\delta=p_{\mathbb{C}}(x) \cdot e^{i \mu_{\mathbb{C}}(x)}$. Since $\left(p_{\mathbb{C}} \cdot e^{i \mu_{\mathbb{C}}}\right)_{\alpha}$ and $\left(p_{\mathbb{C}} \cdot e^{i \mu_{\mathbb{C}}}\right)_{\delta}$ are classical subgroup of $\mathcal{G}, x \cdot y \in\left(p_{\mathbb{C}} \cdot e^{i \mu_{\mathbb{C}}}\right)_{\alpha}$ and $x^{-1} \in\left(p_{\mathbb{C}} \cdot e^{i \mu_{\mathbb{C}}}\right)_{\delta}$. Thus,

$$
p_{\mathbb{C}}(x \cdot y) \cdot e^{i \mu_{\mathbb{C}}(x \cdot y)} \geq \alpha=p_{\mathbb{C}}(x) \cdot e^{i \mu_{\mathbb{C}}(x)} \wedge p_{\mathbb{C}}(y) \cdot e^{i \mu_{\mathbb{C}}(y)},
$$

and

$$
p_{\mathbb{C}}\left(x^{-1}\right) \cdot e^{i \mu_{\mathbb{C}}\left(x^{-1}\right)} \geq \delta=p_{\mathbb{C}}(x) \cdot e^{i \mu_{\mathbb{C}}(x)} .
$$

Similarly,

$$
\begin{aligned}
& q_{\mathbb{C}}(x \cdot y) \cdot e^{i v_{\mathbb{C}}(x \cdot y)} \geq q_{\mathbb{C}}(x) \cdot e^{i v_{\mathbb{C}}(x)} \wedge q_{\mathbb{C}}(y) \cdot e^{i v_{\mathbb{C}}(y)}, \\
& q_{\mathbb{C}}\left(x^{-1}\right) \cdot e^{i v_{\mathbb{C}}\left(x^{-1}\right)} \geq q_{\mathbb{C}}(x) \cdot e^{i v_{\mathbb{C}}(x)} .
\end{aligned}
$$

And

$$
\begin{aligned}
& r_{\mathbb{C}}(x \cdot y) \cdot e^{i \omega_{\mathbb{C}}(x \cdot y)} \leq r_{\mathbb{C}}(x) \cdot e^{i \omega_{\mathbb{C}}(x)} \vee r_{\mathbb{C}}(y) \cdot e^{i \omega_{\mathbb{C}}(y)}, \\
& r_{\mathbb{C}}\left(x^{-1}\right) \cdot e^{i \omega_{\mathbb{C}}\left(x^{-1}\right)} \leq r_{\mathbb{C}}(x) \cdot e^{i \omega_{\mathbb{C}}(x)} .
\end{aligned}
$$

So, the conditions of Definition 3.1 are satisfied. Hence $\mathcal{G}$ is a complex neutrosophic subgroup.

\section{Image and Preimage of Complex Neutrosophic Set}

Definition 5.1. Let $f: \mathcal{G}_{1} \longrightarrow \mathcal{G}_{2}$ be a function and $\mathbb{C}_{1}$ and $\mathbb{C}_{2}$ be the complex neutrosophic sets of $\mathcal{G}_{1}$ and $\mathcal{G}_{2}$, respectively. Then the image of a complex neutrosophic set $\mathbb{C}_{1}$ is a complex neutrosophic set of $\mathcal{G}_{2}$ and it is defined as follows:

$$
\begin{aligned}
f\left(\mathbb{C}_{1}\right)(y) & =\left(p_{f}\left(\mathbb{C}_{1}\right)(y) \cdot e^{i \mu_{f}\left(\mathbb{C}_{1}\right)(y)}, q_{f}\left(\mathbb{C}_{1}\right)(y) \cdot e^{i v_{f}\left(\mathbb{C}_{1}\right)(y),}\right. \\
& \left.r_{f}\left(\mathbb{C}_{1}\right)(y) \cdot e^{i \omega_{f}\left(\mathbb{C}_{1}\right)(y)}\right) \\
& =\left(f\left(p_{\mathbb{C}_{1}}\right)(y) \cdot e^{i f\left(\mu_{\mathbb{C}_{1}}\right)(y)}, f\left(q_{\mathbb{C}_{1}}\right)(y) \cdot e^{i f\left(v_{\mathbb{C}_{1}}\right)(y),}\right. \\
& \left.f\left(r_{\mathbb{C}_{1}}\right)(y) \cdot e^{i f\left(\omega_{\mathbb{C}_{1}}\right)(y)}\right), \forall y \in \mathcal{G}_{2}
\end{aligned}
$$

where,

$$
\begin{aligned}
& f\left(p_{\mathbb{C}_{1}}\right)(y) \cdot e^{i f\left(\mu_{\mathbb{C}_{1}}\right)(y)}=\left\{\begin{array}{cl}
\bigvee p_{\mathbb{C}_{1}}(x) \cdot e^{i \mu_{\mathbb{C}_{1}}(x)}, & \text { if } x \in f^{-1}(y) \\
0 & \text { otherwise }
\end{array}\right. \\
& f\left(q_{\mathbb{C}_{1}}\right)(y) \cdot e^{i f\left(v_{\mathbb{C}_{1}}\right)(y)}=\left\{\begin{array}{cl}
\bigvee q_{\mathbb{C}_{1}}(x) \cdot e^{i v_{\mathbb{C}_{1}}(x)}, & \text { if } x \in f^{-1}(y) \\
0 & \text { otherwise }
\end{array}\right. \\
& f\left(r_{\mathbb{C}_{1}}\right)(y) \cdot e^{i f\left(\omega_{\mathbb{C}_{1}}\right)(y)}=\left\{\begin{array}{cl}
\bigwedge r_{\mathbb{C}_{1}}(x) \cdot e^{i \omega_{\mathbb{C}_{1}}(x)}, & \text { if } x \in f^{-1}(y) \\
1 \cdot e^{i 2 \pi} & \text { otherwise }
\end{array} .\right.
\end{aligned}
$$


And the preimage of a complex neutrosophic set $\mathbb{C}_{2}$ is a complex neutrosophic set of $\mathcal{G}_{1}$ and it is defined as follows: for all $x \in \mathcal{G}_{1}$,

$$
\begin{aligned}
f^{-1}\left(\mathbb{C}_{2}\right)(x) & =\left(p_{f^{-1}}\left(\mathbb{C}_{2}\right)(x) \cdot e^{i \mu_{f^{-1}}\left(\mathbb{C}_{2}\right)(x)}, q_{f^{-1}}\left(\mathbb{C}_{2}\right)(x) \cdot e^{i v_{f^{-1}}\left(\mathbb{C}_{2}\right)(x)},\right. \\
& \left.r_{f^{-1}}\left(\mathbb{C}_{2}\right)(x) \cdot e^{i \omega_{f^{-1}}\left(\mathbb{C}_{2}\right)(x)}\right) \\
& =\left(p_{\mathbb{C}_{2}}(f(x)) \cdot e^{i \mu_{\mathbb{C}_{2}}(f(x))}, q_{\mathbb{C}_{2}}(f(x)) \cdot e^{i v_{\mathbb{C}_{2}}(f(x))},\right. \\
& \left.r_{\mathbb{C}_{2}}(f(x)) \cdot e^{i \omega_{\mathbb{C}_{2}}(f(x))}\right) \\
& =\mathbb{C}_{2}(f(x)) .
\end{aligned}
$$

Theorem 5.2. Let $\mathcal{G}_{1}$ and $\mathcal{G}_{2}$ be two groups and $f: \mathcal{G}_{1} \longrightarrow \mathcal{G}_{2}$ be a group homomorphism. If $\mathbb{C}$ is a complex neutrosophic subgroup of $\mathcal{G}_{1}$, then the image of $\mathbb{C}, f(\mathbb{C})$ is a complex neutrosophic subgroup of $\mathcal{G}_{2}$.

Proof: Let $\mathbb{C}$ be a CNSG of $\mathcal{G}_{1}$ and $y_{1}, y_{2} \in \mathcal{G}_{2}$. if $f^{-1}\left(y_{1}\right)=\phi$ or $f^{-1}\left(y_{2}\right)=\phi$, then it is obvious that $f(\mathbb{C})$ is a CNSG of $\mathcal{G}_{2}$. Let us assume that there exist $x_{1}, x_{2} \in \mathcal{G}_{1}$ such that $f\left(x_{1}\right)=y_{1}$ and $f\left(x_{2}\right)=y_{2}$. Since $f$ is a group homomorphism,

$$
\begin{aligned}
f\left(p_{\mathbb{C}}\left(y_{1} \cdot y_{2}^{-1}\right)\right) \cdot e^{i f\left(\mu_{\mathbb{C}}\left(y_{1} \cdot y_{2}^{-1}\right)\right)} & =\bigvee_{y_{1} \cdot y_{2}^{-1}=f(x)} p_{\mathbb{C}}(x) \cdot e^{i \mu_{\mathbb{C}}(x)} \\
& \geq p_{\mathbb{C}}\left(x_{1} \cdot x_{2}^{-1}\right) \cdot e^{i \mu_{\mathbb{C}}\left(x_{1} \cdot x_{2}^{-1}\right)}, \\
f\left(q_{\mathbb{C}}\left(y_{1} \cdot y_{2}^{-1}\right)\right) \cdot e^{i f\left(v_{\mathbb{C}}\left(y_{1} \cdot y_{2}^{-1}\right)\right)} & =\bigvee_{y_{1} \cdot y_{2}^{-1}=f(x)} q_{\mathbb{C}}(x) \cdot e^{i v_{\mathbb{C}}(x)} \\
& \geq q_{\mathbb{C}}\left(x_{1} \cdot x_{2}^{-1}\right) \cdot e^{i v_{\mathbb{C}}\left(x_{1} \cdot x_{2}^{-1}\right)}, \\
f\left(r_{\mathbb{C}}\left(y_{1} \cdot y_{2}^{-1}\right)\right) \cdot e^{i f\left(\omega_{\mathbb{C}}\left(y_{1} \cdot y_{2}^{-1}\right)\right)} & =\bigwedge_{y_{1} \cdot y_{2}^{-1}=f(x)} r_{\mathbb{C}}(x) \cdot e^{i \omega_{\mathbb{C}}(x)} \\
& \leq r_{\mathbb{C}}\left(x_{1} \cdot x_{2}^{-1}\right) \cdot e^{i \omega_{\mathbb{C}}\left(x_{1} \cdot x_{2}^{-1}\right)} .
\end{aligned}
$$

By using the above inequalities let us prove that

$$
\begin{aligned}
& f(\mathbb{C})\left(y_{1} \cdot y_{2}^{-1}\right) \geq f(\mathbb{C})\left(y_{1}\right) \wedge f(\mathbb{C})\left(y_{2}\right) . \\
& f(\mathbb{C})\left(y_{1} \cdot y_{2}^{-1}\right)=\left(f\left(p_{\mathbb{C}}\left(y_{1} \cdot y_{2}^{-1}\right)\right) \cdot e^{i f\left(\mu_{\mathbb{C}}\left(y_{1} \cdot y_{2}^{-1}\right)\right)}, f\left(q_{\mathbb{C}}\left(y_{1} \cdot y_{2}^{-1}\right)\right) \cdot e^{i f\left(v_{\mathbb{C}}\left(y_{1} \cdot y_{2}^{-1}\right)\right)},\right. \\
& \left.f\left(r_{\mathbb{C}}\left(y_{1} \cdot y_{2}^{-1}\right)\right) \cdot e^{i f\left(\omega_{\mathbb{C}}\left(y_{1} \cdot y_{2}^{-1}\right)\right)}\right) \\
& =\left(\bigvee_{y_{1} \cdot y_{2}^{-1}=f(x)} p_{\mathbb{C}}(x) \cdot e^{i \mu_{\mathbb{C}}(x)}, \bigvee_{y_{1} \cdot y_{2}^{-1}=f(x)} q_{\mathbb{C}}(x) \cdot e^{i v_{\mathbb{C}}(x)},\right. \\
& \left.\bigwedge_{y_{1} \cdot y_{2}^{-1}=f(x)} r_{\mathbb{C}}(x) \cdot e^{i \omega_{\mathbb{C}}(x)}\right) \\
& \geq\left(p_{\mathbb{C}}\left(x_{1} \cdot x_{2}^{-1}\right) \cdot e^{i \mu_{\mathbb{C}}\left(x_{1} \cdot x_{2}^{-1}\right)}, q_{\mathbb{C}}\left(x_{1} \cdot x_{2}^{-1}\right) \cdot e^{i v_{\mathbb{C}}\left(x_{1} \cdot x_{2}^{-1}\right)},\right. \\
& \left.r_{\mathbb{C}}\left(x_{1} \cdot x_{2}^{-1}\right) \cdot e^{i \omega_{\mathcal{C}}\left(x_{1} \cdot x_{2}^{-1}\right)}\right)
\end{aligned}
$$




$$
\begin{aligned}
& \geq\left(p_{\mathbb{C}}\left(x_{1}\right) \cdot e^{i \mu_{\mathbb{C}}\left(x_{1}\right)} \wedge p_{\mathbb{C}}\left(x_{2}\right) \cdot e^{i \mu_{\mathbb{C}}\left(x_{2}\right)}, q_{\mathbb{C}}\left(x_{1}\right) \cdot e^{i v_{\mathbb{C}}\left(x_{1}\right)}\right. \\
& \left.\wedge q_{\mathbb{C}}\left(x_{2}\right) \cdot e^{i v_{\mathbb{C}}\left(x_{2}\right)}, r_{\mathbb{C}}\left(x_{1}\right) \cdot e^{i \omega_{\mathbb{C}}\left(x_{1}\right)} \vee r_{\mathbb{C}}\left(x_{2}\right) \cdot e^{i \omega_{\mathbb{C}}\left(x_{2}\right)}\right) \\
& =\left(p_{\mathbb{C}}\left(x_{1}\right) \cdot e^{i \mu_{\mathbb{C}}\left(x_{1}\right)}, q_{\mathbb{C}}\left(x_{1}\right) \cdot e^{i v_{\mathbb{C}}\left(x_{1}\right)}, r_{\mathbb{C}}\left(x_{1}\right) \cdot e^{i \omega_{\mathbb{C}}\left(x_{1}\right)}\right. \\
& \left.\wedge p_{\mathbb{C}}\left(x_{2}\right) \cdot e^{i \mu_{\mathbb{C}}\left(x_{2}\right)}, q_{\mathbb{C}}\left(x_{2}\right) \cdot e^{i v_{\mathbb{C}}\left(x_{2}\right)}, r_{\mathbb{C}}\left(x_{2}\right) \cdot e^{i \omega_{\mathbb{C}}\left(x_{2}\right)}\right) \\
& =f(\mathbb{C})\left(y_{1}\right) \wedge f(\mathbb{C})\left(y_{2}\right) .
\end{aligned}
$$

This is satisfied for each $x_{1}, x_{2} \in \mathcal{G}_{1}$ with $f\left(x_{1}\right)=y_{1}$ and $f\left(x_{2}\right)=y_{2}$, then it is obvious that

$$
\begin{gathered}
f(\mathbb{C})\left(y_{1} \cdot y_{2}^{-1}\right) \geq \bigvee_{y_{1}=f\left(x_{1}\right)} p_{\mathbb{C}}\left(x_{1}\right) \cdot e^{i \mu_{\mathbb{C}}\left(x_{1}\right)}, \bigvee_{y_{1}=f\left(x_{1}\right)} q_{\mathbb{C}}\left(x_{1}\right) \cdot e^{i v_{\mathbb{C}}\left(x_{1}\right)}, \\
\left.\bigwedge_{y_{1}=f\left(x_{1}\right)} r_{\mathbb{C}}\left(x_{1}\right) \cdot e^{i \omega_{\mathbb{C}}\left(x_{1}\right)}\right) \wedge\left(\bigvee_{y_{2}=f\left(x_{2}\right)} p_{\mathbb{C}}\left(x_{2}\right) \cdot e^{i \mu_{\mathbb{C}}\left(x_{2}\right)},\right. \\
\left.\bigvee_{y_{2}=f\left(x_{2}\right)} q_{\mathbb{C}}\left(x_{2}\right) \cdot e^{i v_{\mathbb{C}}\left(x_{2}\right)}, \bigwedge_{y_{2}=f\left(x_{2}\right)} r_{\mathbb{C}}\left(x_{2}\right) \cdot e^{i \omega_{\mathbb{C}}\left(x_{2}\right)}\right) \\
=\left(f\left(p_{\mathbb{C}}\left(y_{1}\right)\right) \cdot e^{i f\left(\mu_{\mathbb{C}}\left(y_{1}\right)\right)}, f\left(q_{\mathbb{C}}\left(y_{1}\right)\right) \cdot e^{i f\left(v_{\mathbb{C}}\left(y_{1}\right)\right)}, f\left(r_{\mathbb{C}}\left(x_{1}\right)\right) \cdot e^{i f\left(\omega_{\mathbb{C}}\left(x_{1}\right)\right)}\right) \\
\wedge\left(f\left(p_{\mathbb{C}}\left(y_{2}\right)\right) \cdot e^{i f\left(\mu_{\mathbb{C}}\left(y_{2}\right)\right)}, f\left(q_{\mathbb{C}}\left(y_{2}\right)\right) \cdot e^{i f\left(v_{\mathbb{C}}\left(y_{2}\right)\right)}, f\left(r_{\mathbb{C}}\left(x_{2}\right)\right) \cdot e^{i f\left(\omega_{\mathbb{C}}\left(x_{2}\right)\right)}\right) \\
=f(\mathbb{C})\left(y_{1}\right) \wedge f(\mathbb{C})\left(y_{2}\right) .
\end{gathered}
$$

Hence the image of a CNSG is also a CNSG.

Theorem 5.3. Let $\mathcal{G}_{1}$ and $\mathcal{G}_{2}$ be the two groups and $f: \mathcal{G}_{1} \longrightarrow \mathcal{G}_{2}$ be a group homomorphism. If $\mathbb{C}_{2}$ is a complex neutrosophic subgroup of $\mathcal{G}_{2}$, then the preimage of $f^{-1}\left(\mathbb{C}_{2}\right)$ is a complex neutrosophic subgroup of $\mathcal{G}_{1}$.

Proof: Let $\mathbb{C}_{2}$ be a complex neutrosophic subgroup of $\mathcal{G}_{2}$, and $x_{1}, x_{2} \in \mathcal{G}_{1}$. Since $f$ is a group homomorphism, the following inequalities is obtained.

$$
\begin{aligned}
& f^{-1}\left(\mathbb{C}_{2}\right)\left(x_{1} \cdot x_{2}^{-1}\right)=\left(p_{\mathbb{C}_{2}}\left(f\left(x_{1} \cdot x_{2}^{-1}\right)\right) \cdot e^{i \mu_{\mathbb{C}_{2}}\left(f\left(x_{1} \cdot x_{2}^{-1}\right)\right)},\right. \\
& q_{\mathbb{C}_{2}}\left(f\left(x_{1} \cdot x_{2}^{-1}\right)\right) \cdot e^{i v_{\mathbb{C}_{2}}}\left(f\left(x_{1} \cdot x_{2}^{-1}\right)\right), \\
& \left.r_{\mathbb{C}_{2}}\left(f\left(x_{1} \cdot x_{2}^{-1}\right)\right) \cdot e^{i \omega_{\mathbb{C}_{2}}\left(f\left(x_{1} \cdot x_{2}^{-1}\right)\right)}\right) \\
& =\left(p_{\mathbb{C}_{2}}\left(f\left(x_{1}\right) \cdot f\left(x_{2}\right)^{-1}\right) \cdot e^{i \mu_{\mathbb{C}_{2}}}\left(f\left(x_{1}\right) \cdot f\left(x_{2}\right)^{-1}\right),\right. \\
& q_{\mathbb{C}_{2}}\left(f\left(x_{1}\right) \cdot f\left(x_{2}\right)^{-1}\right) \cdot e^{i v_{\mathbb{C}_{2}}\left(f\left(x_{1}\right) \cdot f\left(x_{2}\right)^{-1}\right)} \text {, } \\
& \left.r_{\mathbb{C}_{2}}\left(f\left(x_{1}\right) \cdot f\left(x_{2}\right)^{-1}\right) \cdot e^{i \omega_{\mathbb{C}_{2}}\left(f\left(x_{1}\right) \cdot f\left(x_{2}\right)^{-1}\right)}\right) \\
& \geq\left(p_{\mathbb{C}_{2}}\left(f\left(x_{1}\right) \wedge f\left(x_{2}\right)\right) \cdot e^{i \mu_{\mathbb{C}_{2}}\left(f\left(x_{1}\right) \wedge f\left(x_{2}\right)\right)},\right. \\
& q_{\mathbb{C}_{2}}\left(f\left(x_{1}\right) \wedge f\left(x_{2}\right)\right) \cdot e^{i v_{\mathbb{C}_{2}}\left(f\left(x_{1}\right) \wedge f\left(x_{2}\right)\right)}, \\
& \left.r_{\mathbb{C}_{2}}\left(f\left(x_{1}\right) \vee f\left(x_{2}\right)\right) \cdot e^{i \omega_{\mathbb{C}_{2}}\left(f\left(x_{1}\right) \vee f\left(x_{2}\right)\right)}\right) \\
& =\left(p_{\mathbb{C}_{2}}\left(f\left(x_{1}\right)\right) \cdot e^{i \mu_{\mathbb{C}_{2}}\left(f\left(x_{1}\right)\right)}, q_{\mathbb{C}_{2}}\left(f\left(x_{1}\right) \cdot e^{i v_{\mathbb{C}_{2}}\left(f\left(x_{1}\right)\right)},\right.\right. \\
& r_{\mathbb{C}_{2}}\left(f\left(x_{1}\right) \cdot e^{i \omega_{\mathbb{C}_{2}}\left(f\left(x_{1}\right)\right)}\right) \wedge\left(p_{\mathbb{C}_{2}}\left(f\left(x_{2}\right)\right) \cdot e^{i \mu_{\mathbb{C}_{2}}\left(f\left(x_{2}\right)\right)},\right. \\
& q_{\mathbb{C}_{2}}\left(f\left(x_{2}\right) \cdot e^{i v_{\mathbb{C}_{2}}\left(f\left(x_{2}\right)\right)}, r_{\mathbb{C}_{2}}\left(f\left(x_{2}\right) \cdot e^{i \omega_{\mathbb{C}_{2}}\left(f\left(x_{2}\right)\right)}\right)\right. \\
& =f^{-1}\left(\mathbb{C}_{2}\right)\left(x_{1}\right) \wedge f^{-1}\left(\mathbb{C}_{2}\right)\left(x_{2}\right) \text {. }
\end{aligned}
$$


Hence $f^{-1}\left(\mathbb{C}_{2}\right)$ is a CNSG of $\mathcal{G}_{1}$.

Theorem 5.4. Let $f: \mathcal{G}_{1} \longrightarrow \mathcal{G}_{2}$ be a homomorphism of groups, $\mathbb{C}$ is a CNSG of $\mathcal{G}_{1}$ and define $\mathbb{C}^{-1}: \mathcal{G}_{1} \longrightarrow$ $[0,1] \cdot e^{i[0,2 \pi]} \times[0,1] \cdot e^{i[0,2 \pi]} \times[0,1] \cdot e^{i[0,2 \pi]}$ as $\mathbb{C}^{-1}(x)=\mathbb{C}\left(x^{-1}\right)$ for arbitrary $x \in \mathcal{G}_{1}$. Then the following properties are valid.

(1) $\mathbb{C}^{-1}$ is a CNSG of $\mathcal{G}_{1}$.

(2) $(f(\mathbb{C}))^{-1}=f\left(\mathbb{C}^{-1}\right)$.

Proof: (1) Let $\mathbb{C}$ is a complex neutrosophic subgroup of $\mathcal{G}_{1}$.

Since $\mathbb{C}^{-1}: \mathcal{G}_{1} \longrightarrow[0,1] \cdot e^{i[0,2 \pi]} \times[0,1] \cdot e^{i[0,2 \pi]} \times[0,1] \cdot e^{i[0,2 \pi]}$.

Let for all $x \in \mathcal{G}_{1}$, this implies that, $\mathbb{C}^{-1}(x)=\left(x_{T}, x_{I}, x_{F}\right)$ where $x_{T} \in[0,1] \cdot e^{i[0,2 \pi]}, x_{I} \in[0,1] \cdot e^{i[0,2 \pi]}$ and $x_{F} \in[0,1] \cdot e^{i[0,2 \pi]}$.

So $\mathbb{C}^{-1}$ is a complex neutrosophic subgroup of $\mathcal{G}_{1}$.

(2) Given that $\mathbb{C}^{-1}(x)=\mathbb{C}\left(x^{-1}\right) \forall x \in \mathcal{G}_{1}$.

Since $f: \mathcal{G}_{1} \longrightarrow \mathcal{G}_{2}$ be a homomorphism. As $\mathbb{C}$ is a CNSG of $\mathcal{G}_{1}$ this implies that $\mathbb{C}^{-1}$ is a CNSG of $\mathcal{G}_{1}$ by part $(1)$, so $f\left(\mathbb{C}^{-1}\right) \in \mathcal{G}_{2}$ and $f(\mathbb{C}) \in \mathcal{G}_{2}$. Now by $(1),(f(\mathbb{C}))^{-1} \in \mathcal{G}_{2}$ as $\mathcal{G}_{2}$ is a group homomorphism.

So $f\left(\mathbb{C}^{-1}\right)=(f(\mathbb{C}))^{-1}$ by uniqueness of inverse of an element.

Corollary 5.5. Let $f: \mathcal{G}_{1} \longrightarrow \mathcal{G}_{2}$ be an isomorphism on of groups, $\mathbb{C}$ is complex neutrosophic subgroup of $\mathcal{G}_{1}$, then $f^{-1}(f(\mathbb{C}))=\mathbb{C}$.

Corollary 5.6. Let $f: \mathcal{G} \longrightarrow \mathcal{G}$ be an isomorphism on a group $\mathcal{G}, \mathbb{C}$ is complex neutrosophic subgroup of $\mathcal{G}$, then $f(\mathbb{C})=\mathbb{C}$ if and only if $f^{-1}(\mathbb{C})=\mathbb{C}$.

\section{Complex Neutrosophic Normal Subgroup}

Definition 6.1. Let $\mathbb{C}$ be a complex neutrosophic subgroup of a group $\mathcal{G}$ is known as a complex neutrosophic normal subgroup (CNNSG) of $\mathcal{G}$, if

$\mathbb{C}\left(x y x^{-1}\right) \geq \mathbb{C}(y)$ i.e.,

(i) $p_{\mathbb{C}}\left(x y x^{-1}\right) \cdot e^{i \mu_{\mathbb{C}}\left(x y x^{-1}\right)} \geq p_{\mathbb{C}}(y) \cdot e^{i \mu_{\mathbb{C}}(y)}$

(ii) $q_{\mathbb{C}}\left(x y x^{-1}\right) \cdot e^{i v_{\mathbb{C}}\left(x y x^{-1}\right)} \geq q_{\mathbb{C}}(y) \cdot e^{i v_{\mathbb{C}}(y)}$

(iii) $r_{\mathbb{C}}\left(x y x^{-1}\right) \cdot e^{i \omega_{\mathbb{C}}\left(x y x^{-1}\right)} \leq r_{\mathbb{C}}(y) \cdot e^{i \omega_{\mathbb{C}}(y)}, \forall x, y \in \mathcal{G}$.

Example 6.2. Let $\mathcal{G}=S_{3}=\left\{1, a, a^{2}, b, a b, a^{2} b\right\}$ be a group and $\mathbb{C}=\left\langle T_{\mathbb{C}}, I_{\mathbb{C}}, F_{\mathbb{C}}\right\rangle$ be a complex neutrosophic set of $\mathcal{G}$ such that,

$$
\begin{aligned}
& T_{\mathbb{C}}(1)=0.8 e^{0.6 \pi i}, T_{\mathbb{C}}(a)=T_{\mathbb{C}}\left(a^{2}\right)=0.6 e^{0.6 \pi i} \\
& T_{\mathbb{C}}(b)=T_{\mathbb{C}}(a b)=T_{\mathbb{C}}\left(a^{2} b\right)=0.5 e^{0.4 \pi i} \\
& I_{\mathbb{C}}(1)=0.7 e^{0.5 \pi i}, I_{\mathbb{C}}(a)=I_{\mathbb{C}}\left(a^{2}\right)=0.6 e^{0.5 \pi i} \\
& I_{\mathbb{C}}(b)=I_{\mathbb{C}}(a b)=I_{\mathbb{C}}\left(a^{2} b\right)=0.4 e^{0.3 \pi i} \\
& F_{\mathbb{C}}(1)=0.5 e^{0.4 \pi i}, F_{\mathbb{C}}(a)=F_{\mathbb{C}}\left(a^{2}\right)=0.3 e^{0.2 \pi i} \\
& F_{\mathbb{C}}(b)=F_{\mathbb{C}}(a b)=F_{\mathbb{C}}\left(a^{2} b\right)=0.3 e^{0.2 \pi i}
\end{aligned}
$$

Then clearly $\mathbb{C}$ is a complex neutrosophic normal subgroup of $\mathcal{G}$. 
Theorem 6.3. If $\mathbb{C}_{1}$ and $\mathbb{C}_{2}$ are any two complex neutrosophic normal subgroups of the groups $\mathcal{G}_{1}$ and $\mathcal{G}_{2}$ respectively, then $\mathbb{C}_{1} \times \mathbb{C}_{2}$ is also a complex neutrosophic normal subgroup of $\mathcal{G}_{1} \times \mathcal{G}_{2}$.

Proof: Similarly to the proof of Theorem 3.5.

Theorem 6.4. Let $\mathcal{G}$ be a group, and $\mathbb{C}_{1}$ and $\mathbb{C}_{2}$ be two $C N N S G$ s of $\mathcal{G}$, then $\mathbb{C}_{1} \cap \mathbb{C}_{2}$ is also a complex neutrosophic normal subgroup of $\mathcal{G}$.

Proof: Since $\mathbb{C}_{1}$ and $\mathbb{C}_{2}$ are $C N N S G$ s of $\mathcal{G}$, then

$$
p_{\mathbb{C}_{1}}\left(x \cdot y \cdot x^{-1}\right) \cdot e^{i \mu_{\mathbb{C}_{1}}\left(x \cdot y \cdot x^{-1}\right)} \geq p_{\mathbb{C}_{1}}(y) \cdot e^{i \mu_{\mathbb{C}_{1}}(y)},
$$

and

$$
p_{\mathbb{C}_{2}}\left(x \cdot y \cdot x^{-1}\right) \cdot e^{i \mu_{\mathbb{C}_{2}}\left(x \cdot y \cdot x^{-1}\right)} \geq p_{\mathbb{C}_{2}}(y) \cdot e^{i \mu_{\mathbb{C}_{2}}(y)} .
$$

So, by the definition of the intersection,

$$
\begin{aligned}
p_{\mathbb{C}_{1} \cap \mathbb{C}_{2}}\left(x \cdot y \cdot x^{-1}\right) \cdot e^{i \mu_{\mathbb{C}_{1} \cap \mathbb{C}_{2}}\left(x \cdot y \cdot x^{-1}\right)} & =p_{\mathbb{C}_{1}}\left(x \cdot y \cdot x^{-1}\right) \cdot e^{i \mu_{\mathbb{C}_{1}}\left(x \cdot y \cdot x^{-1}\right)} \\
& \wedge p_{\mathbb{C}_{2}}\left(x \cdot y \cdot x^{-1}\right) \cdot e^{i \mu_{\mathbb{C}_{2}}\left(x \cdot y \cdot x^{-1}\right)} \\
& \geq p_{\mathbb{C}_{1}}(y) \cdot e^{i \mu_{\mathbb{C}_{1}}(y)} \wedge p_{\mathbb{C}_{2}}(y) \cdot e^{i \mu_{\mathbb{C}_{2}}(y)} \\
& =p_{\mathbb{C}_{1} \cap \mathbb{C}_{2}}(y) \cdot e^{i \mu_{\mathbb{C}_{1} \cap \mathbb{C}_{2}}(y)} .
\end{aligned}
$$

By the similar way,

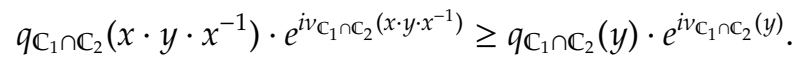

And

$$
\begin{aligned}
r_{\mathbb{C}_{1} \cup \mathbb{C}_{2}}\left(x \cdot y \cdot x^{-1}\right) \cdot e^{i \omega_{\mathbb{C}_{1} \cup \mathbb{C}_{2}}\left(x \cdot y \cdot x^{-1}\right)} & =r_{\mathbb{C}_{1}}\left(x \cdot y \cdot x^{-1}\right) \cdot e^{i \omega_{\mathbb{C}_{1}}\left(x \cdot y \cdot x^{-1}\right)} \\
& \vee r_{\mathbb{C}_{2}}\left(x \cdot y \cdot x^{-1}\right) \cdot e^{i \omega_{\mathbb{C}_{2}}\left(x \cdot y \cdot x^{-1}\right)} \\
& \leq r_{\mathbb{C}_{1}}(y) \cdot e^{i \omega_{\mathbb{C}_{1}}(y)} \vee r_{\mathbb{C}_{2}}(y) \cdot e^{i \omega_{\mathbb{C}_{2}}(y)} \\
& =r_{\mathbb{C}_{1} \cup \mathbb{C}_{2}}(y) \cdot e^{i \omega_{\mathbb{C}_{1} \cup \mathbb{C}_{2}}(y)}
\end{aligned}
$$

Hence the intersection of two CNNSGs is also a CNNSG.

Theorem 6.5. If $\mathbb{C}_{1}$ and $\mathbb{C}_{2}$ be two CNNSGs of $\mathcal{G}$, then $\mathbb{C}_{1} \cup \mathbb{C}_{2}$ is a complex neutrosophic normal subgroup of $\mathcal{G}$.

Proof: Similarly to the proof of Theorem 3.11 .

Proposition 6.6. Let $\mathbb{C}$ be a complex neutrosophic subgroup of a group $\mathcal{G}$. Then the following are correspondent:

(1) $\mathbb{C}$ is a CNNSG of $\mathcal{G}$.

(2) $\mathbb{C}\left(x \cdot y \cdot x^{-1}\right)=\mathbb{C}(y), \forall x, y \in G$.

(3) $\mathbb{C}(x \cdot y)=\mathbb{C}(y \cdot x), \forall x, y \in \mathcal{G}$.

Proof: $(1) \Rightarrow(2)$ : Let $\mathbb{C}$ be a complex neutrosophic normal subgroup of $\mathcal{G}$. Take $x, y \in \mathcal{G}$, then by Definition 6.1 ,

$$
\begin{aligned}
& p_{\mathbb{C}}\left(x \cdot y \cdot x^{-1}\right) \cdot e^{i \mu_{\mathbb{C}}\left(x \cdot y \cdot x^{-1}\right)} \geq p_{\mathbb{C}}(y) \cdot e^{i \mu_{\mathbb{C}}(y)}, \\
& q_{\mathbb{C}}\left(x \cdot y \cdot x^{-1}\right) \cdot e^{i v_{\mathbb{C}}\left(x \cdot y \cdot x^{-1}\right)} \geq q_{\mathbb{C}}(y) \cdot e^{i v_{\mathbb{C}}(y)}, \\
& r_{\mathbb{C}}\left(x \cdot y \cdot x^{-1}\right) \cdot e^{i \omega_{\mathbb{C}}\left(x \cdot y \cdot x^{-1}\right)} \leq r_{\mathbb{C}}(y) \cdot e^{i \omega_{\mathbb{C}}(y)} .
\end{aligned}
$$


Thus taking arbitrary element $x$, the following is got for the truth membership of $\mathbb{C}$,

$$
\begin{aligned}
p_{\mathbb{C}}\left(x^{-1} \cdot y \cdot x\right) \cdot e^{i \mu_{\mathbb{C}}\left(x^{-1} \cdot y \cdot x\right)} & =p_{\mathbb{C}}\left(x^{-1} \cdot y \cdot\left(x^{-1}\right)^{-1} \cdot e^{i \mu_{\mathbb{C}}\left(x^{-1} \cdot y \cdot\left(x^{-1}\right)^{-1}\right)}\right. \\
& \geq p_{\mathbb{C}}(y) \cdot e^{i \mu_{\mathbb{C}}(y)} .
\end{aligned}
$$

Therefore,

$$
\begin{aligned}
p_{\mathbb{C}}(y) \cdot e^{i \mu_{\mathbb{C}}(y)} & =p_{\mathbb{C}}\left(x^{-1} \cdot\left(x \cdot y \cdot x^{-1}\right) \cdot x\right) \cdot e^{i \mu_{\mathbb{C}}\left(x^{-1} \cdot\left(x \cdot y \cdot x^{-1}\right) \cdot x\right)} \\
& \geq p_{\mathbb{C}}\left(x \cdot y \cdot x^{-1}\right) \cdot e^{i \mu_{\mathbb{C}}\left(x \cdot y \cdot x^{-1}\right)}
\end{aligned}
$$

Thus, $p_{\mathbb{C}}\left(x \cdot y \cdot x^{-1}\right) \cdot e^{i \mu_{\mathbb{C}}\left(x \cdot y \cdot x^{-1}\right)}=p_{\mathbb{C}}(y) \cdot e^{i \mu_{\mathbb{C}}(y)}$.

Similarly, $q_{\mathbb{C}}\left(x \cdot y \cdot x^{-1}\right) \cdot e^{i v_{\mathbb{C}}\left(x \cdot y \cdot x^{-1}\right)}=q_{\mathbb{C}}(y) \cdot e^{i v_{\mathbb{C}}(y)}$.

For falsity membership,

$$
\begin{aligned}
r_{\mathbb{C}}\left(x^{-1} \cdot y \cdot x\right) \cdot e^{i \omega_{\mathbb{C}}\left(x^{-1} \cdot y \cdot x\right)} & =r_{\mathbb{C}}\left(x^{-1} \cdot y \cdot\left(x^{-1}\right)^{-1}\right) \cdot e^{i \omega_{\mathbb{C}}\left(x^{-1} \cdot y \cdot\left(x^{-1}\right)^{-1}\right)} \\
& \leq r_{\mathbb{C}}(y) \cdot e^{i \omega_{\mathbb{C}}(y)} .
\end{aligned}
$$

Therefore,

$$
\begin{aligned}
r_{\mathbb{C}}(y) \cdot e^{i \omega_{\mathbb{C}}(y)} & =r_{\mathbb{C}}\left(x^{-1} \cdot\left(x \cdot y \cdot x^{-1}\right) \cdot x\right) \cdot e^{i \omega_{\mathbb{C}}\left(x^{-1} \cdot\left(x \cdot y \cdot x^{-1}\right) \cdot x\right)} \\
& \leq r_{\mathbb{C}}\left(x \cdot y \cdot x^{-1}\right) \cdot e^{i \omega_{\mathbb{C}}\left(x \cdot y \cdot x^{-1}\right)} .
\end{aligned}
$$

This implies that

$$
r_{\mathbb{C}}\left(x \cdot y \cdot x^{-1}\right) \cdot e^{i \omega_{\mathbb{C}}\left(x \cdot y \cdot x^{-1}\right)}=r_{\mathbb{C}}(y) \cdot e^{i \omega_{\mathbb{C}}(y)} .
$$

Hence $\mathbb{C}\left(x \cdot y \cdot x^{-1}\right)=\mathbb{C}(y)$ for all $x, y \in \mathcal{G}$.

(2) $\Rightarrow(3)$ : Substituting $y=y \cdot x$ in (2), the condition (3) is shown easily.

(3) $\Rightarrow(1)$ : According to $\mathbb{C}(y \cdot x)=\mathbb{C}(x \cdot y)$, the equality

$$
\mathbb{C}\left(x \cdot y \cdot x^{-1}\right)=\mathbb{C}\left(y \cdot x \cdot x^{-1}\right)=\mathbb{C}(y) \geq \mathbb{C}(y)
$$

is satisfied. Hence $\mathbb{C}$ is a CNNSG of $\mathcal{G}$.

Theorem 6.7. Let $\mathbb{C}$ is a complex neutrosophic subgroup of a group $\mathcal{G}$. Then $\mathbb{C}$ is a complex neutrosophic normal subgroup of $\mathcal{G}$ if and only if for arbitrary $\alpha=\beta e^{i \gamma}$ where $\beta \in[0,1], \gamma \in[0,2 \pi]$, if $\alpha$-level sets of $\mathbb{C}$ are non-empty, then $\left(p_{\mathbb{C}} \cdot e^{i \mu_{\mathbb{C}}}\right)_{\alpha},\left(q_{\mathbb{C}} \cdot e^{i v_{\mathbb{C}}}\right)_{\alpha}$ and $\left(r_{\mathbb{C}} \cdot e^{i \omega_{\mathbb{C}}}\right)^{\alpha}$ are classical subgroups of $\mathcal{G}$.

Proof: Similarly to the proof of Proposition 4.3.

Theorem 6.8. Let $\mathbb{C}$ is a complex neutrosophic normal subgroup of a group $\mathcal{G}$. Let $\mathcal{G}_{\mathbb{C}}=\left\{x \in \mathcal{G} \mid \mathbb{C}(x) e^{i \mathbb{C}(x)}=\right.$ $\left.\mathbb{C}(\hat{e}) e^{i \mathbb{C}(\hat{e})}\right\}$, where $\hat{e}$ is the unit of $\mathcal{G}$. Then the classical subset $\mathcal{G}_{\mathbb{C}}$ of $\mathcal{G}$ is a normal subgroup of $\mathcal{G}$.

Proof: Let $\mathbb{C}$ be a CNNSG of $\mathcal{G}$. First it is necessary to show that the classical subset $\mathcal{G}_{\mathbb{C}}$ is a subgroup of $\mathcal{G}$. Let us take $x, y \in \mathcal{G}_{\mathbb{C}}$, then by Theorem 3.7,

$$
\begin{aligned}
\mathbb{C}\left(x \cdot y^{-1}\right) e^{i \mathbb{C}\left(x \cdot y^{-1}\right)} & \geq \mathbb{C}(x) e^{i \mathbb{C}(x)} \wedge \mathbb{C}(y) e^{i \mathbb{C}(y)} \\
& =\mathbb{C}(\hat{e}) e^{i \mathbb{C}(\hat{e})} \wedge \mathbb{C}(\hat{e}) e^{i \mathbb{C}(\hat{e})} \\
& =\mathbb{C}(\hat{e}) e^{i \mathbb{C}(\hat{e})}
\end{aligned}
$$


and always $\mathbb{C}(\hat{e}) e^{i \mathbb{C}(\hat{e})} \geq \mathbb{C}\left(x \cdot y^{-1}\right) e^{i \mathbb{C}\left(x \cdot y^{-1}\right)}$.

Hence $x \cdot y^{-1} \in \mathcal{G}_{\mathbb{C}}$, i.e., $\mathcal{G}_{\mathbb{C}}$ is a subgroup of $\mathcal{G}$.

Now we will be shown that $\mathcal{G}_{\mathbb{C}}$ is normal. Take arbitrary $x \in \mathcal{G}_{\mathbb{C}}$ and $y \in \mathcal{G}$. Therefore, $\mathbb{C}(x) e^{i \mathbb{C}(x)}=\mathbb{C}(\hat{e}) e^{i \mathbb{C}(\hat{e})}$. Since $\mathbb{C} \in \operatorname{CNNSG}(\mathcal{G})$, the following is obtained,

$$
\begin{aligned}
\mathbb{C}\left(y \cdot x \cdot y^{-1}\right) e^{i \mathbb{C}\left(y \cdot x \cdot y^{-1}\right)} & =\mathbb{C}\left(y^{-1} \cdot y \cdot x\right) e^{i \mathbb{C}\left(y^{-1} \cdot y \cdot x\right)} \\
& =\mathbb{C}(x) e^{i \mathbb{C}(x)}=\mathbb{C}(\hat{e}) e^{i \mathbb{C}(\hat{e})} .
\end{aligned}
$$

Hence, $y \cdot x \cdot y^{-1} \in \mathcal{G}_{\mathbb{C}}$, So $\mathcal{G}_{\mathbb{C}}$ is a normal subgroup of $\mathcal{G}$.

Theorem 6.9. Let $f: \mathcal{G}_{1} \longrightarrow \mathcal{G}_{2}$ be a group homomorphism and $\mathbb{C}_{2}$ is a $C N N S G$ of $\mathcal{G}_{2}$. Then the preimage $f^{-1}\left(\mathbb{C}_{2}\right)$ is a $C N N S G$ of $\mathcal{G}_{1}$.

Proof: From the Theorem 5.3, it is known that $f^{-1}\left(\mathbb{C}_{2}\right)$ is a complex neutrosophic subgroup of $\mathcal{G}_{1}$. Hence it is sufficient to show that normality property of $f^{-1}\left(\mathbb{C}_{2}\right)$. For arbitrary $x_{1}, x_{2} \in \mathcal{G}_{1}$, by homomorphism of $f$ and by the normality of $\mathbb{C}_{2}$,

$$
\begin{aligned}
f^{-1}\left(\mathbb{C}_{2}\right)\left(x_{1} \cdot x_{2}\right) e^{i f^{-1}\left(\mathbb{C}_{2}\right)\left(x_{1} \cdot x_{2}\right)} & =\mathbb{C}_{2}\left(f\left(x_{1} \cdot x_{2}\right)\right) e^{i \mathbb{C}_{2}\left(f\left(x_{1} \cdot x_{2}\right)\right)} \\
& =\mathbb{C}_{2}\left(f\left(x_{1}\right) \cdot f\left(x_{2}\right)\right) e^{i \mathbb{C}_{2}\left(f\left(x_{1}\right) \cdot f\left(x_{2}\right)\right)} \\
& =\mathbb{C}_{2}\left(f\left(x_{2}\right) \cdot f\left(x_{1}\right)\right) e^{i \mathbb{C}_{2}\left(f\left(x_{2}\right) \cdot f\left(x_{1}\right)\right)} \\
& =\mathbb{C}_{2}\left(f\left(x_{2} \cdot x_{1}\right)\right) e^{i \mathbb{C}_{2}\left(f\left(x_{2} \cdot x_{1}\right)\right)} \\
& =f^{-1}\left(\mathbb{C}_{2}\right)\left(x_{2} \cdot x_{1}\right) e^{i f^{-1}\left(\mathbb{C}_{2}\right)\left(x_{2} \cdot x_{1}\right)} .
\end{aligned}
$$

Hence, from the Proposition 6.6, $f^{-1}\left(\mathbb{C}_{2}\right)$ is a CNNSG of $\mathcal{G}_{1}$.

Theorem 6.10. Let $f: \mathcal{G}_{1} \longrightarrow \mathcal{G}_{2}$ be a surjective homomorphism of groups $\mathcal{G}_{1}$ and $\mathcal{G}_{2}$. if $\mathbb{C}$ is a $C N N S G$ of $\mathcal{G}_{1}$, then $f(\mathbb{C})$ is a CNNSG of $\mathcal{G}_{2}$.

Proof: Since $f(\mathbb{C})$ is a complex neutrosophic subgroup of $\mathcal{G}_{2}$ is clear from the Theorem 5.2, it is sufficient only to show that the normality condition by using Proposition 6.6 (3). Take $y_{1}, y_{2} \in \mathcal{G}_{2}$ such that $f^{-1}\left(y_{1}\right) \neq \phi$, $f^{-1}\left(y_{2}\right) \neq \phi$ and $f^{-1}\left(y_{1} \cdot y_{2}^{-1}\right) \neq \phi$. So it is inferred that

$$
f\left(p_{\mathbb{C}}\left(y_{1} \cdot y_{2} \cdot y_{1}^{-1}\right)\right) e^{i f\left(\mu_{\mathbb{C}}\left(y_{1} \cdot y_{2} \cdot y_{1}^{-1}\right)\right)}=\bigvee_{l \in f^{-1}\left(y_{1} \cdot y_{2} \cdot y_{1}^{-1}\right)} p_{\mathbb{C}}(l) e^{i \mu_{\mathbb{C}}(l)}
$$

and

$$
f\left(p_{\mathbb{C}}\left(y_{2}\right)\right) e^{i f\left(\mu_{\mathbb{C}}\left(y_{2}\right)\right)}=\bigvee_{l \in f^{-1}\left(y_{2}\right)} p_{\mathbb{C}}(l) e^{i \mu_{\mathbb{C}}(l)} .
$$

For all $x_{2} \in f^{-1}\left(y_{2}\right), x_{1} \in f^{-1}\left(y_{1}\right)$ and $x_{1}^{-1} \in f^{-1}\left(y_{1}^{-1}\right)$, since $\mathbb{C}$ is normal,

$$
\begin{aligned}
& p_{\mathbb{C}}\left(x_{1} \cdot x_{2} \cdot x_{1}^{-1}\right) e^{i \mu_{\mathbb{C}}\left(x_{1} \cdot x_{2} \cdot x_{1}^{-1}\right)} \geq p_{\mathbb{C}}\left(x_{2}\right) e^{i \mu_{\mathbb{C}}\left(x_{2}\right)}, \\
& q_{\mathbb{C}}\left(x_{1} \cdot x_{2} \cdot x_{1}^{-1}\right) e^{i \nu_{\mathbb{C}}\left(x_{1} \cdot x_{2} \cdot x_{1}^{-1}\right)} \geq q_{\mathbb{C}}\left(x_{2}\right) e^{i \nu_{\mathbb{C}}\left(x_{2}\right)}, \\
& r_{\mathbb{C}}\left(x_{1} \cdot x_{2} \cdot x_{1}^{-1}\right) e^{i \omega_{\mathbb{C}}\left(x_{1} \cdot x_{2} \cdot x_{1}^{-1}\right)} \leq r_{\mathbb{C}}\left(x_{2}\right) e^{i \omega_{\mathbb{C}}\left(x_{2}\right)}
\end{aligned}
$$

are obtained.

Since $f$ is a homomorphism, it follows that

$$
f\left(x_{1} \cdot x_{2} \cdot x_{1}^{-1}\right)=f\left(x_{1}\right) \cdot f\left(x_{2}\right) \cdot f\left(x_{1}\right)^{-1}=y_{1} \cdot y_{2} \cdot y_{1}^{-1} .
$$


So, $x_{1} \cdot x_{2} \cdot x_{1}^{-1} \in f^{-1}\left(y_{1} \cdot y_{2} \cdot y_{1}^{-1}\right)$. Hence

$$
\begin{aligned}
\bigvee_{l \in f^{-1}\left(y_{1} \cdot y_{2} \cdot y_{1}^{-1}\right)} p_{\mathbb{C}}(l) e^{i \mu_{\mathbb{C}}(l)} & \geq \underset{x_{1} \in f^{-1}\left(y_{1}\right), x_{2} \in f^{-1}\left(y_{2}\right)}{ } p_{\mathbb{C}}\left(x_{1} \cdot x_{2} \cdot x_{1}^{-1}\right) e^{i \mu_{\mathbb{C}}\left(x_{1} \cdot x_{2} \cdot x_{1}^{-1}\right)} \\
& \geq \bigvee_{x_{2} \in f^{-1}\left(y_{2}\right)} p_{\mathbb{C}}\left(x_{2}\right) e^{i \mu_{\mathbb{C}}\left(x_{2}\right)}
\end{aligned}
$$

This means that,

$$
f\left(p_{\mathbb{C}}\left(y_{1} \cdot y_{2} \cdot y_{1}^{-1}\right)\right) e^{i f\left(\mu_{\mathbb{C}}\left(y_{1} \cdot y_{2} \cdot y_{1}^{-1}\right)\right)} \geq f\left(p_{\mathbb{C}}\left(y_{2}\right)\right) e^{i f\left(\mu_{\mathbb{C}}\left(y_{2}\right)\right)} .
$$

On the other hand, the following inequalities are obtained in a similar observation.

$$
\begin{aligned}
& f\left(q_{\mathbb{C}}\left(y_{1} \cdot y_{2} \cdot y_{1}^{-1}\right)\right) e^{i f\left(v_{\mathbb{C}}\left(y_{1} \cdot y_{2} \cdot y_{1}^{-1}\right)\right)} \geq f\left(q_{\mathbb{C}}\left(y_{2}\right)\right) e^{i f\left(v_{\mathbb{C}}\left(y_{2}\right)\right)}, \\
& f\left(r_{\mathbb{C}}\left(y_{1} \cdot y_{2} \cdot y_{1}^{-1}\right)\right) e^{i f\left(\omega_{\mathbb{C}}\left(y_{1} \cdot y_{2} \cdot y_{1}^{-1}\right)\right)} \geq f\left(r_{\mathbb{C}}\left(y_{2}\right)\right) e^{i f\left(\omega_{\mathbb{C}}\left(y_{2}\right)\right)} .
\end{aligned}
$$

So the desired inequality,

$$
\begin{aligned}
f(\mathbb{C})\left(y_{1} \cdot y_{2} \cdot y_{1}^{-1}\right) e^{i f(\mathbb{C})\left(y_{1} \cdot y_{2} \cdot y_{1}^{-1}\right)} & =\left(f\left(p_{\mathbb{C}}\left(y_{1} \cdot y_{2} \cdot y_{1}^{-1}\right)\right) e^{i f\left(\mu_{\mathbb{C}}\left(y_{1} \cdot y_{2} \cdot y_{1}^{-1}\right)\right),}\right. \\
& f\left(q_{\mathbb{C}}\left(y_{1} \cdot y_{2} \cdot y_{1}^{-1}\right)\right) e^{i f\left(v_{\mathbb{C}}\left(y_{1} \cdot y_{2} \cdot y_{1}^{-1}\right)\right)}, \\
& \left.f\left(r_{\mathbb{C}}\left(y_{1} \cdot y_{2} \cdot y_{1}^{-1}\right)\right) e^{i f\left(\omega_{\mathbb{C}}\left(y_{1} \cdot y_{2} \cdot y_{1}^{-1}\right)\right)}\right) \\
& \geq\left(f\left(p_{\mathbb{C}}\left(y_{2}\right)\right) e^{i f\left(\mu_{\mathbb{C}}\left(y_{2}\right)\right)}, f\left(q_{\mathbb{C}}\left(y_{2}\right)\right) e^{i f\left(v_{\mathbb{C}}\left(y_{2}\right)\right)},\right. \\
& \left.f\left(r_{\mathbb{C}}\left(y_{2}\right)\right) e^{i f\left(\omega_{\mathbb{C}}\left(y_{2}\right)\right)}\right) \\
& =\left(p_{f(\mathbb{C})}\left(y_{2}\right) e^{i \mu_{f(\mathbb{C}}\left(y_{2}\right)}, q_{f(\mathbb{C})}\left(y_{2}\right) e^{i v_{f(\mathbb{C})}\left(y_{2}\right)},\right. \\
& \left.r_{f(\mathbb{C})}\left(y_{2}\right) e^{i \omega_{f(\mathbb{C}}\left(y_{2}\right)}\right) \\
& =f(\mathbb{C})\left(y_{2}\right) e^{i f(\mathbb{C})\left(y_{2}\right)},
\end{aligned}
$$

is satisfied.

\section{Conclusion}

In this paper we presented the concept of complex neutrosophic subgroups (normal subgroups) and alpha-cut of complex neutrosophic set, and studied some of its motivating results. We have also defined the Cartesian product of complex neutrosophic subgroups and discussed some its related results. Furthermore, we have also defined the concept of image and preimage of complex neutrosophic set and studied some of its properties. In future, we will generalized the study to soft set theory and will initiate the concept of soft complex neutrosophic subgroups (normal subgroups).

\section{Acknowledgment}

The authors would like to thank the reviewers for their valuable comments and helpful suggestions for improvement of the original manuscript. 


\section{References}

[1] Zadeh, L. A. 1965. Fuzzy Sets. Inform and Control, 8; 338-353.

[2] Atanassov, K. T. 1986. Intuitionistic Fuzzy Sets, Fuzzy Sets and Systems, 20; 87-96.

[3] Rosenfeld, A. 1971. Fuzzy Groups. J. Math. Anal. Appl., 35; 512-517.

[4] Wang, H. et al., 2005. Single Valued Neutrosophic Sets. Proc of 10th Int Conf on Fuzzy Theory and Technology, Salt Lake City, Utah.

[5] Smarandache, F. 1999. A Unifying Field in Logics. Neutrosophy: Neutrosophic Probability, Set and logic, Rehoboth; American Research Press.

[6] Buckley, J. J. 1989. Fuzzy Complex Numbers. Fuzzy Sets and Systems. 33; 333-345.

[7] Nguyen, H. T. Kandel, A. and Kreinovich, V. 2000. Complex Fuzzy Sets. Towards New Foundations, IEEE. 7803-5877.

[8] Ramot, D. Milo, R. Friedman, M. Kandel, A. 2002. Complex fuzzy sets. IEEE Transactionson Fuzzy Systems. 10; 171-186.

[9] Zhang, G. Dillon, T. S. Cai, K. Y. Ma, J. and Lu, J. 2009. Operation Properties and $\delta$-Equalities of Complex Fuzzy Sets. International Journal of Approximate Reasoning, 50; 1227-1249.

[10] Abd Ulazeez, M. Alkouri, S. and Salleh, A. R. 2012. Complex Intuitionistic Fuzzy Sets. International Conference on Fundamental and Applied Sciences, AIP Conference Proceedings, 1482; 464-470.

[11] Abd Ulazeez, M. Alkouri, S. and Salleh, A. R. 2013. Complex Atanassov's Intuitionistic Fuzzy Relation. Hindawi Publishing Corporation Abstract and Applied Analysis, Volume 2013, Article ID 287382, 18pages.

[12] Ali, M and Smarandache, F. 2016. Complex Neutrosophic Set. Neural Comput \& Applic. DOI 10.1007/s00521-015-2154-y.

[13] Cetkin, V. and Aygun, H. 2017. An Approach to Neutrosophic Subgroup and its Fundamental Properties. Journal of Intelligent \& Fuzzy Systems, (In Press).

[14] Turksen, I. B. (1986). Interval-Valued Fuzzy Sets Based on Normal Forms. Fuzzy Sets and Systems 20; 191-210.

[15] Yaqoob, N. Akram, M. and Aslam, M. (2013). Intuitionistic fuzzy soft groups induced by (t,s)-norm. Indian Journal of Science and Technology 6(4); 4282-4289. 